\title{
Three-nucleon forces from chiral effective field theory
}

\author{
E. Epelbaum, ${ }^{1, *}$ A. Nogga, ${ }^{2, \dagger}$ W. Glöckle, ${ }^{1, \$}$ H. Kamada, ${ }^{3, \S}$ Ulf-G. Meißner ${ }^{4, \|}$ and H. Witała ${ }^{5, \pi}$ \\ ${ }^{1}$ Ruhr-Universität Bochum, Institut für Theoretische Physik II, D-44870 Bochum, Germany \\ ${ }^{2}$ Department of Physics, University of Arizona, Tucson, Arizona 85721 \\ ${ }^{3}$ Department of Physics, Faculty of Engineering, Kyushu Institute of Technology, 1-1 Sensuicho, Tobata, Kitakyushu 804-8550, Japan \\ ${ }^{4}$ Forschungszentrum Jülich, Institut für Kernphysik (Theorie), D-52425 Jülich, Germany \\ and Karl-Franzens-Universität Graz, Institut für Theoretische Physik A-8010 Graz, Austria \\ ${ }^{5}$ Jagiellonian University, Institute of Physics, Reymonta 4, 30-059 Cracow, Poland
}

(Received 14 August 2002; published 11 December 2002)

\begin{abstract}
We perform the first fully consistent analysis of $n d$ scattering at next-to-next-to-leading order in chiral effective field theory including the corresponding three-nucleon force and extending our previous work, where only the two-nucleon interaction has been taken into account. The three-nucleon force appears first at this order in the chiral expansion and depends on two unknown parameters. These two parameters are determined from the triton binding energy and $n d$ doublet scattering length. We find an improved description of various scattering observables in relation to the next-to-leading order results especially at moderate energies $\left(E_{\text {lab }}\right.$ $=65 \mathrm{MeV}$ ). It is demonstrated that the long-standing $A_{y}$ problem in $n d$ elastic scattering is still not solved by the leading $3 \mathrm{NF}$, although some visible improvement is observed. We discuss possibilities of solving this puzzle. The predicted binding energy for the $\alpha$ particle agrees with the empirical value.
\end{abstract}

DOI: 10.1103/PhysRevC.66.064001

PACS number(s): 21.45.+v, 21.30.-x, 27.10.+h, 25.10.+s

\section{INTRODUCTION}

Effective field theory has become a standard tool for analyzing the chiral structure of quantum chromodynamics (QCD) at low energy, where the perturbative expansion in powers of the coupling constant cannot be used. The chiral symmetry of QCD is spontaneously broken and the corresponding Goldstone bosons can be identified with pions, if one considers the two flavor sector of the up and down quarks as done here. The pions are not exactly massless as it would be the case for massless $u$ and $d$ quarks, but are much lighter than all other hadrons and are therefore sometimes called pseudo-Goldstone bosons. It is a general property of Goldstone bosons that their interactions become weak for small momenta. Chiral perturbation theory (CHPT) is an effective field theory which allows to describe the interactions of pions and between pions and matter fields (nucleons, $\rho$-mesons, $\Delta$-resonances, $\ldots$ ) in a systematic way. This is achieved via an expansion of the scattering amplitude in powers of small external momenta and the pion mass. Pion loops are naturally incorporated and all corresponding ultraviolet divergences can be absorbed at each fixed order in the chiral expansion by counter terms of the most general chiral invariant Lagrangian.

This perturbative scheme works well in the pion and pionnucleon sector, where the interaction vanishes at vanishing external momenta in the chiral limit. The situation in the purely nucleonic sector is somewhat different, since the in-

\footnotetext{
*Electronic address: evgeni.epelbaum@tp2.ruhr-uni-bochum.de

${ }^{\dagger}$ Electronic address: anogga@physics.arizona.edu

\#Electronic address: walter.gloeckle@tp2.ruhr-uni-bochum.de

${ }^{\S}$ Electronic address: kamada@mns.kyutech.ac.jp

"Electronic address: u.meissner@fz-juelich.de

II Electronic address: witala@if.uj.edu.pl
}

teraction between nucleons is strong and remains strong even in the chiral limit at vanishing three-momenta of the external nucleons. The main difficulty in the direct application of the standard methods of CHPT to the nucleon-nucleon $(N N)$ system is due to the nonperturbative aspect of the problem. One way to deal with this difficulty has been suggested by Weinberg, who proposed to apply CHPT to the kernel of the corresponding integral equation for the scattering amplitude, which can be viewed as an effective $N N$ potential [1,2].

Following this idea Weinberg was able to demonstrate the validity of the well-established intuitive hierarchy of the few-nucleon forces: the two-nucleon interactions are more important than the three-nucleon ones, which are more important than the four-nucleon interactions and so on.

The first quantitative realization of the above idea has been performed by Ordónez and co-workers, who derived the $2 \mathrm{~N}$ potential and performed a numerical analysis of the two-nucleon system [3]. To calculate an expression for the effective Hamiltonian for two nucleons the authors of [3] made use of Rayleigh-Schrödinger perturbation theory (the method is closely related to the Tamm-Dancoff approach $[4,5])$, which leads to a non-Hermitian and energy-dependent potential. The $\Delta$-degree of freedom has been included explicitely. The 26 free parameters, many of them being redundant due to the property of antisymmetry of the wave functions, have been fixed from a global fit to the low-energy observables. Ordóñez et al. obtained qualitative fits to deuteron properties as well as quantitative fits to most of the scattering phase shifts up to $E_{\mathrm{lab}}=100 \mathrm{MeV}$.

The property of the effective potential from Ref. [3] of being explicitly energy-dependent makes it difficult to apply it to systems different from the two-nucleon one. In fact, such an energy dependence is not a fundamental feature of the effective interaction and can be eliminated by certain techniques, see, e.g., [6]. In [7] we have demonstrated how to 
derive the energy-independent and Hermitian potential from the chiral Lagrangian using the method of unitary transformation [8]. The advantage of this scheme is that it is easily extendable to processes with more than two nucleons and/or external fields. In [9] we applied the above mentioned method to calculate the $N N$ scattering observables and deuteron properties up to NNLO in the chiral expansion. As described in Ref. [10], the nine unknown low-energy constants (LECs) related to contact interactions and the LECs $c_{3}$ and $c_{4}$ related to the subleading $\pi \pi N N$ vertices have been fixed by a fit to the Nijmegen phase shifts [11] in the ${ }^{1} S_{0}$, ${ }^{3} S_{1}-{ }^{3} D_{1},{ }^{1} P_{1},{ }^{3} P_{0},{ }^{3} P_{1},{ }^{3} P_{2}-{ }^{3} F_{2}$ channels below $E_{\text {lab }}$ $=100 \mathrm{MeV}$. In contrast to Ref. [3] we did not perform a global fit to the data, which due to the large dimension of the parameter space and computational resource limitations might not lead to the true global minimum in the $\chi^{2}$-space and cannot easily be performed. Instead we introduced an alternative set of partial-wave projected LECs and considered each of the above indicated channels separately having at most three unknown parameters in any given partial wave. The chiral potential at NNLO has been shown to lead to a reasonably good description of the $\mathrm{NN}$ phase shifts up to $E_{\mathrm{lab}} \sim 200 \mathrm{MeV}$ as well as of the deuteron properties. Further we demonstrated that including the subleading two-pion exchange at NNLO allows to improve strongly the NLO results without introducing additional free parameters associated with short-range contact interactions, which is a good indication of consistency and convergence of the chiral expansion. For our choice of the LECs $c_{1,3,4}$ related to the subleading $\pi \pi N N$ interactions see Ref. [10]. The corresponding $N N$ potential has been denoted in [10] by NNLO*. The LEC's $c_{i}$ chosen in [10] had the values $c_{1}=-0.81 \mathrm{GeV}^{-1}, c_{3}$ $=-1.15 \mathrm{GeV}^{-1}$, and $c_{4}=1.20 \mathrm{GeV}^{-1}$. These have to be compared with the values extracted from various analyses of $\pi N$ scattering at leading and next-to-leading order: $c_{1}$ $=-0.64$ to $-1.53 \mathrm{GeV}^{-1}, c_{3}=-3.90$ to $-6.19 \mathrm{GeV}^{-1}$, and $c_{4}=2.25$ to $4.12 \mathrm{GeV}^{-1}$. As explained in [10], using average values in these ranges results in a very strong attractive two-pion exchange potential and, as a consequence, spurious $N N$ bound states appear in the low partial waves. The latter do not lead to any harm in the $N N$ system (since they are outside the range of the validity of the EFT description) but cause technical difficulties in systems with more than two nucleons. The NNLO* potential with numerically smaller values of $c_{3,4}$ given above leads to an equally good description of the $N N$ phase shifts as the one with the larger values resulting from $\pi N$ scattering but is free from the spurious deeply bound states. Although some arguments have been given in [10] in favor of the smaller values of these low-energy constants, a better understanding of the link between $\pi N$ scattering and $N N$ two-pion exchange is needed. In this sense the NNLO* potential should be considered as a preliminary step. In a future investigation we are going to study the role of regularization in generating the very strong TPE, which might shed some light on that problem. Here in this paper we stick to that choice of NNLO*, drop, however, the star for simplicity.

The few-nucleon interactions in chiral effective field theory have been first discussed qualitatively by Weinberg
[2]. The corresponding expressions have been derived later by van Kolck, who demonstrated that the leading contribution, which appears at NLO in the chiral expansion of the Schrödinger equation kernel, cancels against the iteration of the energy-dependent part of the corresponding $N N$ effective potential [12]. Such a cancellation in case of the two-pion exchange 3NF has already been observed earlier [13]. Thus the first nonvanishing contribution to the $3 \mathrm{NF}$ appears at NNLO. Note that if the $\Delta$-resonance is included explicitly, i.e., if the $\Delta N$ mass splitting is considered as a small quantity of the order of the pion mass, the nonvanishing contributions to the $3 \mathrm{NF}$ are shifted to NLO. Note, however, that such a scheme is not strictly rooted in QCD because of the decoupling theorem [14] (but can be justified in the large $N_{c}$-expansion, with $N_{c}$ the number of colors). For the energy-independent potential derived with the method of unitary transformation one observes the vanishing of the NLO 3NF as well (as has also been pointed out in a different context, e.g., in Ref. [15]) and the first nonvanishing contributions appear at NNLO.

In our work [16] we performed a complete analysis of the low-energy $n d$ scattering at NLO in the chiral expansion with the $N N$ potential introduced in [9] and also calculated the triton and $\alpha$-particle binding energies (BEs). Since no $3 \mathrm{NF}$ has to be included at this order and all parameters in the $N N$ potential are fixed from the $2 N$ system, the results for $A>2$ systems are parameter-free predictions. We demonstrated a reasonably good description of the $n d$ elastic scattering data at $E_{\mathrm{lab}}=3 \mathrm{MeV}$ and $E_{\mathrm{lab}}=10 \mathrm{MeV}$ as well as of some break-up observables at $E_{\mathrm{lab}}=13 \mathrm{MeV}$ while significant deviations from the data were found at $E_{\mathrm{lab}}=65 \mathrm{MeV}$. The predicted value for the triton $\mathrm{BE}$ is in the range comparable to the one based upon various modern phenomenological potentials, while for the $\alpha$-particle BE somewhat larger deviations have been observed depending on the chosen cutoff value. Extending the analysis to NNLO requires, as already stated before, not only the appropriate modification of the $N N$ interaction, but also the inclusion of the 3NF. In [10] we presented an incomplete NNLO analysis of the $3 N$ system based upon the $N N$ interaction at NNLO and without inclusion of the $3 \mathrm{NF}$ [76]. In this work we present the complete NNLO analysis of the low-energy $n d$ scattering including the chiral 3NF. We also predict the $\alpha$-particle binding energy. This is the first time that the complete chiral 3NF has been included in few-body calculations. Some pioneering steps in that direction based upon the hybrid approach have been done in Ref. [17].

Our paper is organized as follows. In Sec. II we discuss the structure of the chiral $3 \mathrm{NF}$ and demonstrate that it depends on two parameters. The partial-wave decomposition of the new terms in the 3NF is given in the Appendix. In Sec. III we discuss how these unknown parameters can be fixed from low-energy $3 N$ data. Then we show our results for various elastic and break-up $n d$ scattering observables as well as for triton and $\alpha$-particle BEs in Sec. IV. Conclusions and an outlook are given in Sec. V.

\section{THE CHIRAL THREE-NUCLEON FORCE AT NEXT-TO-NEXT-TO-LEADING ORDER}

The chiral $3 \mathrm{NF}$ at NNLO is given by the two-pion exchange (TPE), one-pion exchange (OPE) with the pion emit- 


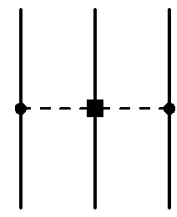

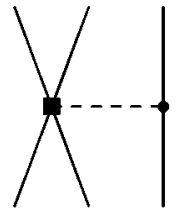

2

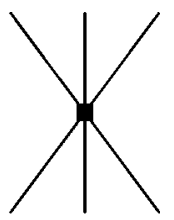

3
FIG. 1. Three-nucleon force at NNLO: TPE, OPE and contact interaction. Solid and dashed lines are nucleons and pions, respectively. Heavy dots denote leading vertices with $\Delta_{i}=0$ and solid rectangles correspond to vertices of dimension $\Delta_{i}=1$.

ted (or absorbed) by $2 \mathrm{~N}$ contact interactions and $3 \mathrm{~N}$ contact interactions, see Fig. 1. All diagrams include apart from the leading vertices with $\Delta_{i}=0$ one insertion of interactions with $\Delta_{i}=1$, where the chiral dimension is defined as

$$
\Delta_{i}=d_{i}+\frac{1}{2} n_{i}-2
$$

Here $d_{i}$ and $n_{i}$ denote the number of derivatives (or pion mass insertions) and nucleon fields for a vertex of type $i$. This quantity has been first introduced by Weinberg and is especially useful in the few-nucleon sector. In the pion and pion-nucleon sectors one usually uses a different definition.

The contribution from the first graph in Fig. 1 is given (in the $3 N \mathrm{cms}$ ) by [12] (here and in what follows we use the usual notation for expressing the nuclear force: the quantity $V_{\mathrm{TPE}}^{3 \mathrm{NF}}$ is an operator with respect to spin and isospin quantum numbers and a matrix element with respect to momentum quantum numbers):

$$
V_{\mathrm{TPE}}^{3 \mathrm{NF}}=\sum_{i \neq j \neq k} \frac{1}{2}\left(\frac{g_{A}}{2 F_{\pi}}\right)^{2} \frac{\left(\vec{\sigma}_{i} \cdot \vec{q}_{i}\right)\left(\vec{\sigma}_{j} \cdot \vec{q}_{j}\right)}{\left(\vec{q}_{i}^{2}+M_{\pi}^{2}\right)\left(\vec{q}_{j}^{2}+M_{\pi}^{2}\right)} F_{i j k}^{\alpha \beta} \tau_{i}^{\alpha} \tau_{j}^{\beta}
$$

where $\vec{q}_{i} \equiv \vec{p}_{i}^{\prime}-\vec{p}_{i} ; \vec{p}_{i}\left(\vec{p}_{i}^{\prime}\right)$ are initial (final) momenta of the nucleon $i$ and

$$
\begin{aligned}
F_{i j k}^{\alpha \beta}= & \delta^{\alpha \beta}\left[-\frac{4 c_{1} M_{\pi}^{2}}{F_{\pi}^{2}}+\frac{2 c_{3}}{F_{\pi}^{2}} \vec{q}_{i} \cdot \vec{q}_{j}\right] \\
& +\sum_{\gamma} \frac{c_{4}}{F_{\pi}^{2}} \epsilon^{\alpha \beta \gamma} \tau_{k}^{\gamma} \vec{\sigma}_{k} \cdot\left[\vec{q}_{i} \times \vec{q}_{j}\right] .
\end{aligned}
$$

Here, $g_{A}=1.26$ is the axial-vector coupling constant, $F_{\pi}$ $=92.4 \mathrm{MeV}$ the weak pion decay constant and the $c_{1,3,4}$ are the LECs from the chiral Lagrangian of the order $\Delta=1$ [18], which also enter the corresponding expressions for the subleading two-pion exchange in the $2 N$ potential. The form (2) can be shown to match with the low-momentum expansion of various existing phenomenological 3NFs provided they respect chiral symmetry. This issue is extensively discussed in [19].

We will now derive the expressions for the OPE and contact parts of the $3 \mathrm{NF}$, see also [12], and show that due to the Pauli principle only one independent OPE term and one independent pure contact term appear in the $3 \mathrm{NF}[77,20]$.
Let us start with the OPE contribution and discuss first the structure of the corresponding $\pi N N N N$-vertex of dimension $\Delta=1$. After performing the nonrelativistic reduction for the nucleon field (or, equivalently, after integrating out the lower components in the heavy-baryon formalism) one encounters three different structures in the effective Lagrangian (in the rest-frame system of the nucleons):

$$
\begin{aligned}
\mathcal{L}^{(1)}= & \alpha_{1}\left(N^{\dagger} N\right)\left(N^{\dagger} \vec{\sigma} \boldsymbol{\tau} N\right) \cdot \vec{\nabla} \boldsymbol{\pi}+\alpha_{2}\left(N^{\dagger} \vec{\sigma} N\right)\left(N^{\dagger} \boldsymbol{\tau} N\right) \cdot \vec{\nabla} \boldsymbol{\pi} \\
& +\alpha_{3}\left(N^{\dagger} \vec{\sigma} \boldsymbol{\tau} N\right) \times\left(N^{\dagger} \vec{\sigma} \boldsymbol{\tau} N\right) \cdot \vec{\nabla} \boldsymbol{\pi},
\end{aligned}
$$

where $\boldsymbol{\pi}$ and $N$ denote the pion and nonrelativistic nucleon fields, $\sigma_{i}$ and $\tau_{i}$ are Pauli spin and isospin matrices. The symbol $\cdot(X)$ denotes the simultaneous scalar (vector) product in the ordinary and isospin-space. Note that the terms with derivatives acting on the nucleon fields are eliminated by partial integration. The corresponding $3 N$ force at NNLO is of the form

$$
\begin{aligned}
V_{\mathrm{OPE}}^{3 \mathrm{NF}} \propto \sum_{i \neq j \neq k}\left(\vec{q}_{k} \cdot \vec{\sigma}_{k}\right) \frac{\vec{q}_{k} \boldsymbol{\tau}_{k}}{\vec{q}_{k}^{2}+M_{\pi}^{2}}\left\{\alpha_{1} \vec{\sigma}_{i} \boldsymbol{\tau}_{i}+\alpha_{2} \vec{\sigma}_{i} \boldsymbol{\tau}_{j}\right. \\
\left.\quad+\alpha_{3}\left(\vec{\sigma}_{i} \times \vec{\sigma}_{j}\right)\left(\boldsymbol{\tau}_{i} \times \boldsymbol{\tau}_{j}\right)\right\} .
\end{aligned}
$$

Since we treat nucleons as identical particles, the fewnucleon states $|\Psi\rangle$ are antisymmetric. For these antisymmetric states the operators $V_{\mathrm{OPE}}^{3 \mathrm{NF}}$ can be further simplified. Because the force is symmetric with respect to an interchange of particles $i$ and $j$, the relation

$$
V_{\mathrm{OPE}}^{3 \mathrm{NF}}|\Psi\rangle=\mathcal{A}_{i j} V_{\mathrm{OPE}}^{3 \mathrm{NF}}|\Psi\rangle=V_{\mathrm{OPE}}^{3 \mathrm{NF}} \mathcal{A}_{i j}|\Psi\rangle
$$

holds and therefore one can work equally well with an antisymmetrized force. Here $\mathcal{A}_{i j}$ is the antisymmetrization operator in the space of two nucleons $i$ and $j$, which reads

$$
\mathcal{A}_{i j}=\frac{1-P_{i j}}{2}
$$

where $P_{i j}$ is the corresponding permutation operator, $P_{i j}|i j\rangle=|j i\rangle$, given by

$$
P_{i j}=\frac{1+\vec{\sigma}_{i} \cdot \vec{\sigma}_{j}}{2} \frac{1+\tau_{i} \cdot \tau_{j}}{2}
$$

In addition, one has to interchange the corresponding nucleon momenta. It is an easy exercise to apply the antisymmetrization operator $\mathcal{A}_{i j}$ to that pair $i j$ of the $3 \mathrm{NF}$ in Eq. (4) which interacts via the contact terms and to see that all three different structures lead to the same expression.

In the case of the purely contact $3 \mathrm{NF}$ without derivatives we proceed in an analogous way. The most general structure of such $3 \mathrm{NF}$ which satisfies the usual symmetry requirements (rotational and isospin invariance, parity invariance and invariance under time reversal transformation) is given by 


$$
\begin{aligned}
V_{\mathrm{cont}}^{3 \mathrm{NF}}= & \sum_{i \neq j \neq k}\left\{\beta_{1}+\beta_{2} \vec{\sigma}_{i} \cdot \vec{\sigma}_{j}+\beta_{3} \boldsymbol{\tau}_{i} \cdot \boldsymbol{\tau}_{j}\right. \\
& +\beta_{4}\left(\vec{\sigma}_{i} \cdot \vec{\sigma}_{j}\right)\left(\boldsymbol{\tau}_{i} \cdot \boldsymbol{\tau}_{j}\right)+\beta_{5}\left(\vec{\sigma}_{i} \cdot \vec{\sigma}_{j}\right)\left(\boldsymbol{\tau}_{j} \cdot \boldsymbol{\tau}_{k}\right) \\
& \left.+\beta_{6}\left(\left[\vec{\sigma}_{i} \times \vec{\sigma}_{j}\right] \cdot \vec{\sigma}_{k}\right)\left(\left[\boldsymbol{\tau}_{i} \times \boldsymbol{\tau}_{j}\right] \cdot \boldsymbol{\tau}_{k}\right)\right\}
\end{aligned}
$$

The antisymmetrization operator $\mathcal{A}_{i j k}$ in the space of three nucleons can be expressed as

$$
\mathcal{A}_{i j k}=\frac{\left(1+P_{i j} P_{j k}+P_{i k} P_{j k}\right)}{3} \frac{\left(1-P_{j k}\right)}{2} .
$$

Acting with the operator $\mathcal{A}_{i j k}$ on the $3 \mathrm{NF}$ in Eq. (8) and performing a straightforward, but somewhat tedious simplification one ends up with a single structure just as in the previously considered case. We thus have shown that it is sufficient to consider only one OPE and one pure contact term in the chiral $3 \mathrm{NF}$ at NNLO, since all other terms have due to the Pauli principle precisely the same effect on the $S$ matrix. In what follows, we will use the following form for these $3 \mathrm{NF}$ contributions:

$$
\begin{gathered}
V_{\mathrm{OPE}}^{3 \mathrm{NF}}=-\sum_{i \neq j \neq k} \frac{g_{A}}{8 F_{\pi}^{2}} D \frac{\vec{\sigma}_{j} \cdot \vec{q}_{j}}{\vec{q}_{j}^{2}+M_{\pi}^{2}}\left(\boldsymbol{\tau}_{i} \cdot \boldsymbol{\tau}_{j}\right)\left(\vec{\sigma}_{i} \cdot \vec{q}_{j}\right), \\
V_{\mathrm{cont}}^{3 \mathrm{NF}}=\frac{1}{2} \sum_{j \neq k} E\left(\boldsymbol{\tau}_{j} \cdot \boldsymbol{\tau}_{k}\right),
\end{gathered}
$$

where $D$ and $E$ are the corresponding LECs from the Lagrangian of dimension $\Delta=1$ :

$$
\begin{aligned}
\mathcal{L}^{(1)}= & -\frac{D}{4 F_{\pi}}\left(N^{\dagger} N\right)\left(N^{\dagger} \vec{\sigma} \boldsymbol{\tau} N\right) \cdot \vec{\nabla} \boldsymbol{\pi} \\
& -\frac{1}{2} E\left(N^{\dagger} N\right)\left(N^{\dagger} \boldsymbol{\tau} N\right) \cdot\left(N^{\dagger} \boldsymbol{\tau} N\right) .
\end{aligned}
$$

Note that dimensional scaling arguments allow one to express the LECs $D$ and $E$ as [21]

$$
D=\frac{c_{D}}{F_{\pi}^{2} \Lambda_{\chi}}, \quad E=\frac{c_{E}}{F_{\pi}^{4} \Lambda_{\chi}},
$$

where $c_{D}$ and $c_{E}$ should be numbers of order one and $\Lambda_{\chi}$ is the chiral symmetry breaking scale of the order of the $\rho$ meson mass. Here and in what follows we use $\Lambda_{\chi}$ $=700 \mathrm{MeV}$. It has been demonstrated in [22] that all corresponding numbers for $2 \mathrm{~N}$ contact interactions at NLO and NNLO are natural for the cutoff values considered. It should also be understood that a more precise analysis of the naturalness would require also taking into account symmetry factors in the Lagrangian as well as additional factors resulting from insertions of spin and isospin matrices [78].

\section{FIXING THE PARAMETERS OF THE THREE-NUCLEON FORCE}

We now proceed to fix the unknown LECs $c_{D}$ and $c_{E}$ from $3 N$ low-energy observables. To that aim we solve the $3 N$ Faddeev equations for the bound state and for $n d$ scattering. They have the well known form $[23,24]$

$$
\psi=G_{0} t P \psi+\left(1+G_{0} t\right) G_{0} V_{3 \mathrm{NF}}^{(1)}(1+P) \psi,
$$

in case of the bound state. Here $V_{3 \mathrm{NF}}^{(1)}$ is that part of the three-nucleon force which singles out one particle (here particle 1) and which is symmetrical under the exchange of the other two particles. The complete $3 \mathrm{NF}$ is decomposed as

$$
V_{3 \mathrm{NF}}=V_{3 \mathrm{NF}}^{(1)}+V_{3 \mathrm{NF}}^{(2)}+V_{3 \mathrm{NF}}^{(3)} .
$$

Further, $\psi$ denotes the corresponding Faddeev component, $t$ is the two-body $t$-operator, $G_{0}=1 /\left(E-H_{0}\right)$ is the free propagator of three nucleons and $P$ is a sum of a cyclical and anticyclical permutation of the three particles. In case of $n d$ scattering we follow our by now standard path $[25,26]$ and first calculate a quantity $T$ related to the $3 N$ break-up process via the Faddeev-type equation:

$$
\begin{aligned}
T= & t P \phi+\left(1+t G_{0}\right) V_{3 \mathrm{NF}}^{(1)}(1+P) \phi+t P G_{0} T \\
& +\left(1+t G_{0}\right) V_{3 \mathrm{NF}}^{(1)}(1+P) G_{0} T,
\end{aligned}
$$

where the initial state $\phi$ is composed of a deuteron and a momentum eigenstate of the projectile nucleon. The elastic $n d$ scattering operator is then obtained as

$$
U=P G_{0}^{-1}+P T+V_{3 \mathrm{NF}}^{(1)}(1+P)\left(1+G_{0} T\right),
$$

and the break-up operator via

$$
U_{0}=(1+P) T
$$

These equations are accurately solved in momentum space using a partial wave decomposition. For details see $[23,27,28]$. The corresponding partial wave decomposition of the chiral $3 \mathrm{NF}$ is given in the Appendix. Equations (13) and (15) have to be regularized, since the expressions for the $3 \mathrm{NF}$ (2) and (10) are only meaningful for momenta below a certain scale. We regularize the $V^{3 \mathrm{NF}}$ in the way analogous to the one adopted in the analysis of the two-nucleon system [9]:

$$
V^{3 \mathrm{NF}}\left(\vec{p}, \vec{q} ; \vec{p}^{\prime}, \vec{q}^{\prime}\right) \rightarrow f_{R}(\vec{p}, \vec{q}) V^{3 \mathrm{NF}}\left(\vec{p}, \vec{q} ; \vec{p}^{\prime}, \vec{q}^{\prime}\right) f_{R}\left(\vec{p}^{\prime}, \vec{q}^{\prime}\right),
$$

where $\vec{p}$ and $\vec{q}\left(\vec{p}^{\prime}\right.$ and $\left.\vec{q}^{\prime}\right)$ are Jacobi momenta of the twobody subsystem and spectator nucleon before (after) the interaction. The regulator function $f_{R}(\vec{p}, \vec{q})$ is chosen in the form

$$
f_{R}(\vec{p}, \vec{q})=\exp \left[-\left(\frac{4 p^{2}+3 q^{2}}{4 \Lambda^{2}}\right)^{2}\right],
$$




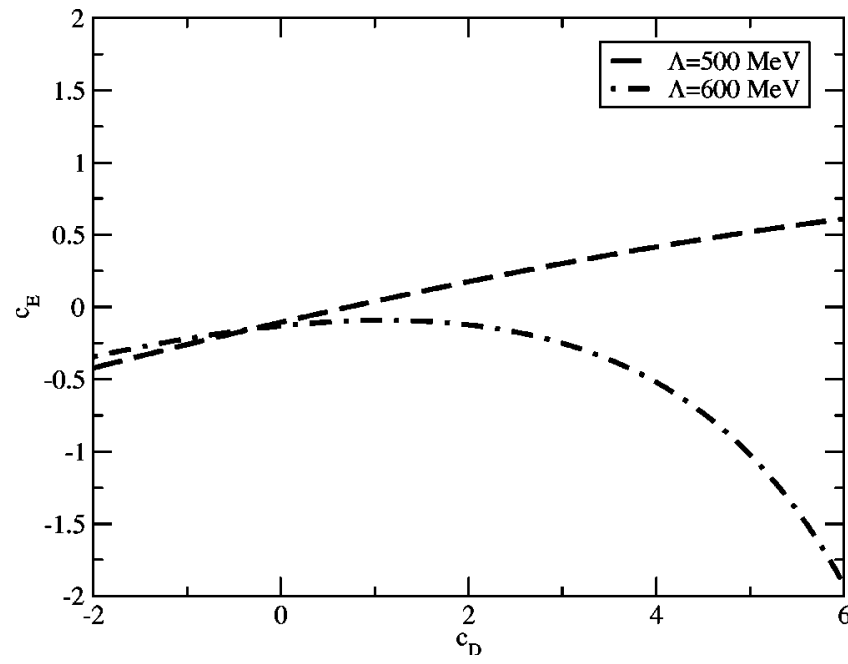

FIG. 2. Correlation between the LECs $c_{E}$ and $c_{D}$ after adjustment to the triton pseudo-BE.

so that it coincides with the exponential function $f_{R}^{\text {expon }}(\vec{p})$ of Ref. [9] for $\vec{q}=0$. Clearly, this is not the only possible choice for that function. The final results for low-energy observables are insensitive to the choice of the regulator function provided that it does not violate the appropriate symmetries. Note that the values of the LECs $c_{D}$ and $c_{E}$ "run" with the cutoff $\Lambda$ to compensate the changes in the observables, which are cutoff independent (up to the accuracy at the order in the chiral expansion). The dependence of the LECs on the cutoff $\Lambda$ is governed by renormalization group equations, as is always the case in quantum field theory. We choose $\Lambda$ in the $3 \mathrm{NF}$ equal to $\Lambda$ in the $N N$ interaction. The following study has been carried through with the minimal and maximal momentum cutoffs, $\Lambda=500$ and $600 \mathrm{MeV}$, for which our $N N$ force has been defined in [10]. In choosing the range of $\Lambda$ we follow closely the strategy suggested by Lepage [29], according to which $\Lambda$ should be large enough to account for relevant long-range physics (in our case one- and two-pion exchanges). Further, one should still stay below the mass scale associated with the states not included in the EFT explicitly. To be more specific, the upper bound $\Lambda$ $=600 \mathrm{MeV}$ results if we require that no unphysical deeply

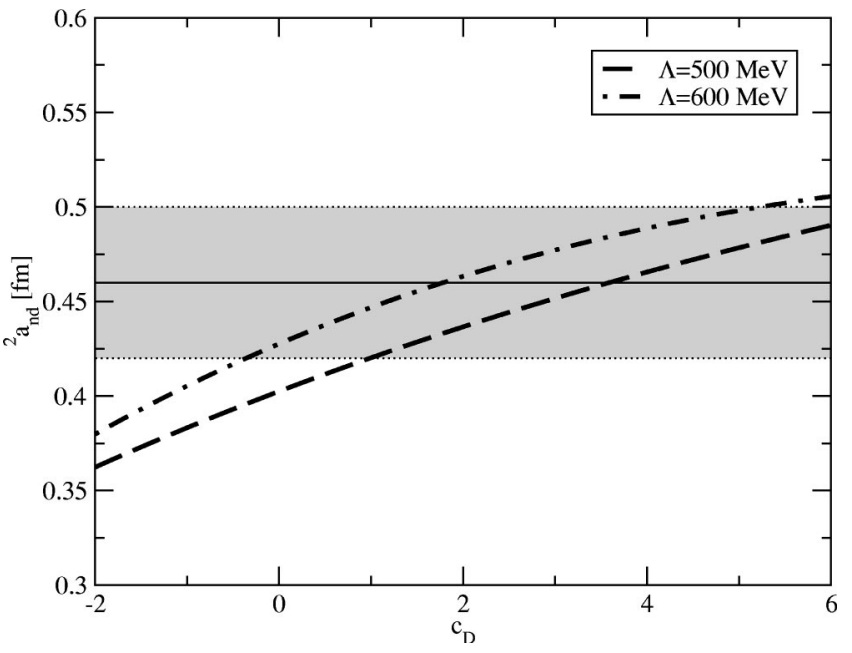

FIG. 3. nd doublet scattering length ${ }^{2} a_{n d}$ as function of the constant $c_{D}$.

bound states appear in low $N N$ partial waves. Although we could, in principle, further reduce the cutoff range below $\Lambda$ $=500 \mathrm{MeV}$, we refrain from doing that since with our choice of the regulator function this would significantly affect observables at moderate energies we are interested in. For example, the $N N$ potential $V(p, p)$ is reduced by $\sim 25 \%$ for $p=306 \mathrm{MeV}$, which corresponds to $E_{\mathrm{lab}} \sim 200 \mathrm{MeV}$ due to multiplication with the regulator function with $\Lambda$ $=500 \mathrm{MeV}$. Notice that such a reduction shows up, e.g., in high partial waves, where the $T$ matrix is essentially given by $V(p, p)$. Obviously, for smaller values of $\Lambda$ the effects of the momentum cutoff become even more dramatic, so that one is restricted to smaller energies. It would be interesting in the future to implement a different regulator function which would allow for a larger variation of $\Lambda$ without such restrictions on the range of applicability of the EFT.

The low-energy constants $c_{D}$ and $c_{E}$ enter the expressions for the chiral $3 \mathrm{NF}$ at NNLO. The constant $c_{E}$ can only be obtained from $3 N$ data, while $c_{D}$ can be best determined in the $3 N$ system or, for larger momentum transfer, in pion production in $N N$ collisions [30]. One important part of this work is to outline a feasible way to fix these parameters. We will now show that the LECs $c_{D}$ and $c_{E}$ can be determined

TABLE I. ${ }^{3} \mathrm{H}$ and ${ }^{4} \mathrm{He} \mathrm{BE}$ at NLO and NNLO of the chiral expansion (for the cutoff range considered throughout) compared to "experimental" pseudo-BE (see text). Apart from the BEs $E$ (in MeV), we also give the kinetic energies $T$ (in $\mathrm{MeV}$ ) as well as expectation values of $2 N$ and $3 N$ forces $V_{N N}$ and $V_{3 N}$, respectively (in MeV).

\begin{tabular}{|c|c|c|c|c|c|c|c|c|c|}
\hline & \multirow[b]{2}{*}{$\Lambda$} & \multicolumn{4}{|c|}{${ }^{3} \mathrm{H}$} & \multicolumn{4}{|c|}{${ }^{4} \mathrm{He}$} \\
\hline & & $E$ & $T$ & $V_{N N}$ & $V_{3 N}$ & $E$ & $T$ & $V_{N N}$ & $V_{3 N}$ \\
\hline \multirow[t]{2}{*}{ NLO } & 500 & -8.54 & 30.76 & -39.30 & & -29.57 & 61.42 & -91.00 & \\
\hline & 600 & -7.53 & 39.24 & -46.77 & & -23.87 & 77.61 & -101.47 & \\
\hline \multirow[t]{2}{*}{ NNLO } & 500 & -8.68 & 31.07 & -39.43 & -0.318 & -29.51 & 61.83 & -89.59 & -1.753 \\
\hline & 600 & -8.68 & 34.44 & -42.41 & -0.712 & -29.98 & 71.49 & -97.44 & -4.025 \\
\hline Expt. & & -8.68 & & & & $-29.8 \pm 0.1$ & & & \\
\hline
\end{tabular}


TABLE II. Contribution of the different terms of the $3 \mathrm{NF}$ to the complete $3 \mathrm{NF}$ expectation value for ${ }^{3} \mathrm{H}$ and ${ }^{4} \mathrm{He}$. All energies are given in $\mathrm{MeV}$.

\begin{tabular}{cccccccccc}
\hline \hline & \multicolumn{1}{c}{} & \multicolumn{2}{c}{${ }^{3} \mathrm{H}$} & \multicolumn{4}{c}{${ }^{4} \mathrm{He}$} \\
& $\Lambda$ & $c$ terms & $D$ term & $E$ term & All & $c$ terms & $D$ term & $E$ term & All \\
\hline \multirow{2}{*}{ NNLO } & 500 & -0.39 & 0.81 & -0.74 & -0.32 & -2.00 & 3.93 & -3.69 & -1.75 \\
& 600 & -0.73 & -0.12 & 0.13 & -0.71 & -3.81 & -0.84 & 0.63 & -4.03 \\
\hline \hline
\end{tabular}

using the ${ }^{3} \mathrm{H} \mathrm{BE}$ and the $n d$ doublet scattering length ${ }^{2} a_{n d}$, which are bona fide low-energy observables. Notice that the choice of low-energy observables used to fix the constants in the $3 \mathrm{NF}$ is by no means unique. The contact force in the second line of Eq. (10) only contributes to the spin-doublet $S$ wave. Therefore, ideally one would like to fix $c_{E}$ from the corresponding phase shift. Equivalently, one can require to reproduce the scattering length in that channel, analogously to what is usually done in the two-nucleon sector. It is more difficult to determine the $\operatorname{LEC} c_{D}$, which contributes to all partial waves. One possible way to proceed would be to perform a $\chi^{2}$ analysis of the low-energy scattering observables. Alternatively, one might chose a different low-energy observable which is sensitive to the unknown LEC as a second constraint. We follow here this second path and take the tri-
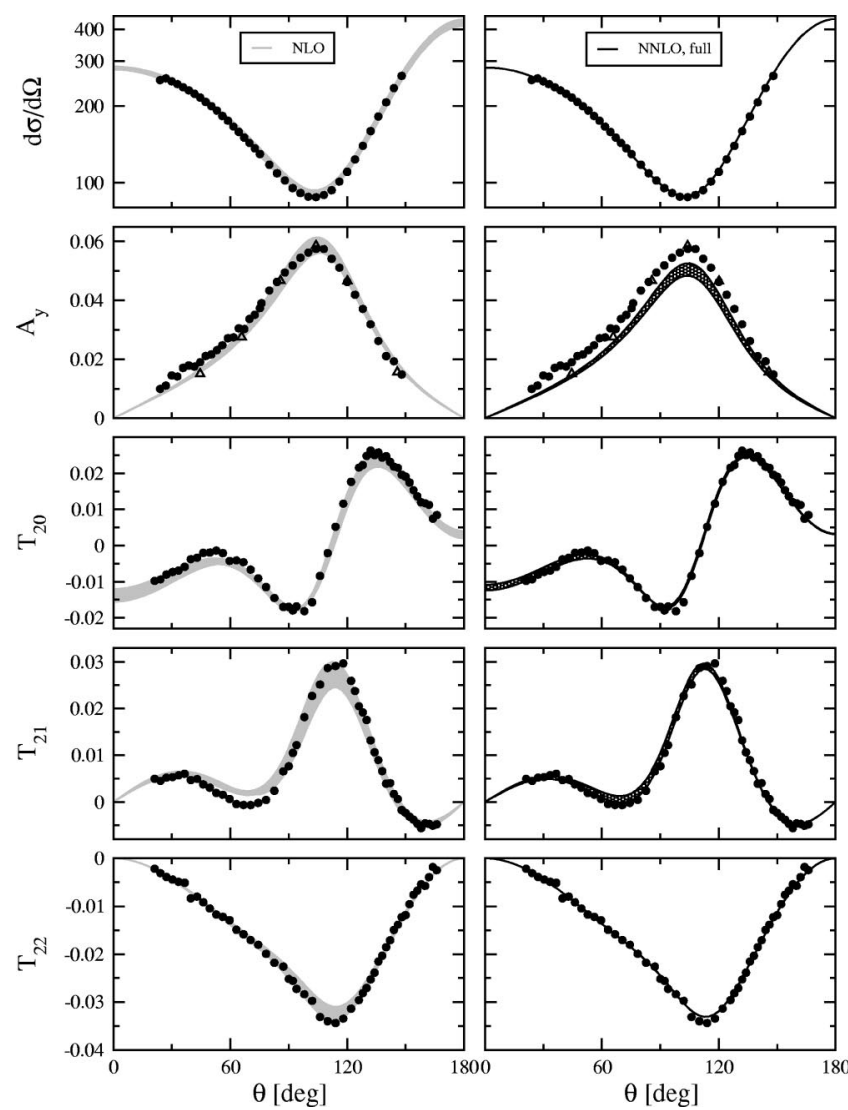

FIG. 4. nd elastic scattering observables at $3 \mathrm{MeV}$ at NLO (left column) and NNLO (right column). The filled circles are $n d$ pseudodata based on $[63,64]$ while the triangles are true $n d$ data [65]. The bands correspond to the cutoff variation between 500 and $600 \mathrm{MeV}$. The unit of the cross section is $\mathrm{mb} / \mathrm{sr}$. ton binding energy as such an observable. Note that one could equally well use, e.g., the $\alpha$-particle binding energy or the differential cross section as the second constraint. We will demonstrate below that our way of fixing the LECs is consistent with the above mentioned observables.

Since for the time being we have no $n n$ and $p p$ forces at our disposal (these have been calculated in chiral EFT to NLO so far [31]) and both observables we are interested in are known to depend on the difference between $n p$ and $n n$ forces, we decided to use $n p / n n$ corrected data as input to our fitting procedure. To this aim, we compare results using phenomenological forces with the proper $n p$ and $n n$ forces and with a $n p$ force only. Several combinations of $N N$ and $3 N$ forces have been adjusted to describe the triton BE (see [32]). We used AV18 [33] augmented by the Urbana-IX 3NF
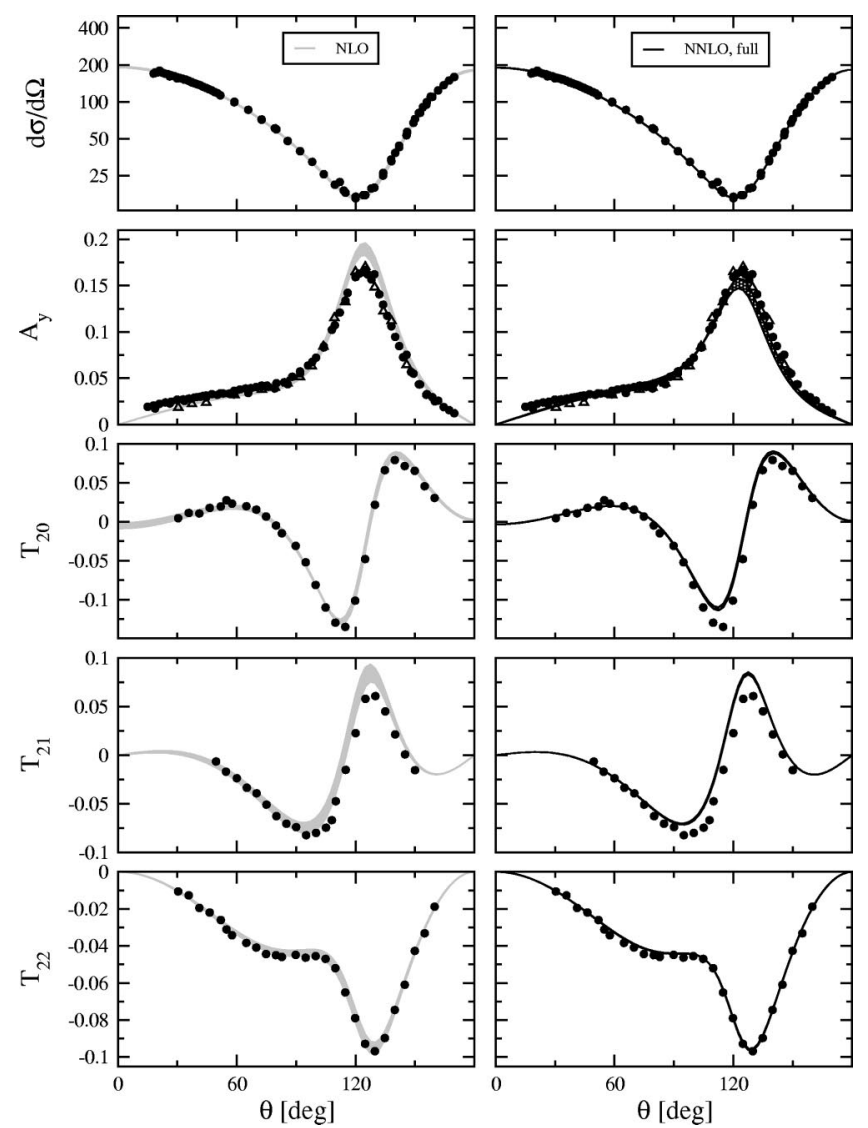

FIG. 5. $n d$ elastic scattering observables at $10 \mathrm{MeV}$ at NLO (left column) and NNLO (right column). The filled circles are $n d$ pseudodata based on $[64,66,67]$ while the triangles are true $n d$ data [68]. The bands correspond to the cutoff variation between 500 and $600 \mathrm{MeV}$. The unit of the cross section is $\mathrm{mb} / \mathrm{sr}$. 

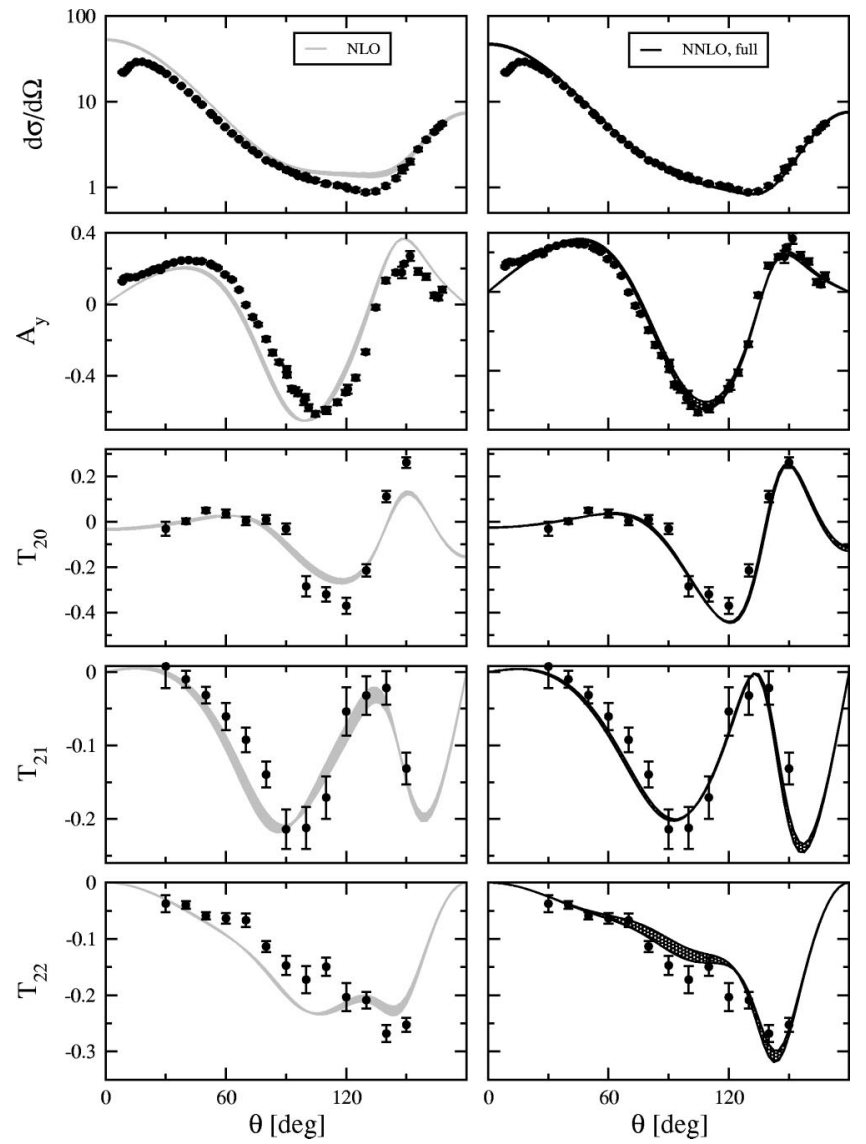

FIG. 6. $n d$ elastic scattering observables at $65 \mathrm{MeV}$ at NLO (left column) and NNLO (right column). The filled circles are $p d$ data $[63,69]$. The bands correspond to the cutoff variation between 500 and $600 \mathrm{MeV}$. The unit of the cross section is $\mathrm{mb} / \mathrm{sr}$.

[34] and CD-Bonn 2000 [35] augmented by the TM99' 3NF [36]. These models come along with $n n$ forces, which are adjusted to the $n n$ scattering length. Replacing these $n n$ forces by the $n p$ ones, we find an increased binding energy of 8.65 and $8.72 \mathrm{MeV}$, respectively. From those we estimate a $n p$ corrected "experimental" pseudo-BE of $8.68 \mathrm{MeV}$ [79].

The "experimental" pseudovalue for ${ }^{2} a_{n d}$ has been determined using the $N N$ force CD-Bonn alone. The corresponding shift of ${ }^{2} a_{n d}$ is $-0.19 \mathrm{fm}$. This together with the experimental value ${ }^{2} a_{n d}=0.64 \pm 0.04 \mathrm{fm}$ leads to the "experimental" pseudovalue ${ }^{2} a_{n d}=0.45 \pm 0.04$. It should be understood that the uncertainty in the estimated pseudovalue of the scattering length is even larger due to the error in the shift resulting from replacement of the $n n$ force by the $n p$ one. We however refrain from further discussion of that issue.

For the chiral interactions at NNLO two unknown LECs enter into the $3 \mathrm{~N}$ bound state Faddeev equation: $c_{D}$ and $c_{E}$. Both affect the BE strongly. Imposing the condition that the Hamiltonian describes the pseudo-BE, we find a correlation between both LECs, which is displayed in Fig. 2. The unsymmetric interval shown in this figure is a consequence of the fact that the doublet scattering length favors positive values for $c_{D}$. The correlations have a very different behavior
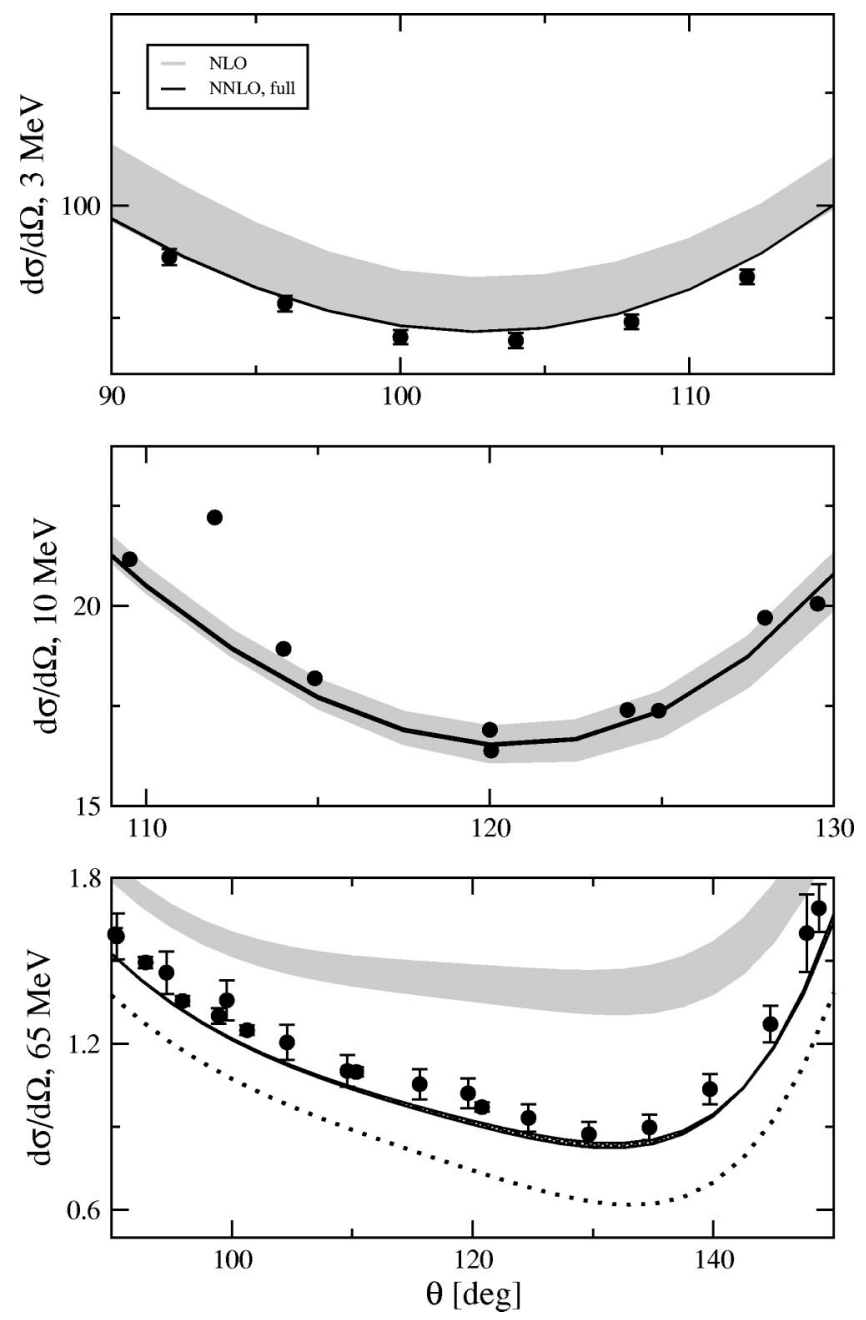

FIG. 7. Minima of the cross section (in $\mathrm{mb} / \mathrm{sr}$ ) of elastic $n d$ scattering at $3 \mathrm{MeV}$ (upper panel), $10 \mathrm{MeV}$ (panel in the middle), and $65 \mathrm{MeV}$ (lower panel) at NLO and NNLO. The filled circles are $n d$ pseudodata at 3 and $10 \mathrm{MeV}$ and true $p d$ data at $65 \mathrm{MeV}$. The bands correspond to the cutoff variation between 500 and $600 \mathrm{MeV}$. The dotted line at $65 \mathrm{MeV}$ shows the NNLO result with $c_{D}$ $=-3.0$ and $\Lambda=500 \mathrm{MeV}$.

for both cutoff values. For $\Lambda=500 \mathrm{MeV}$ the functional form turns out to be nearly linear. This is not the case for $\Lambda$ $=600 \mathrm{MeV}$. Later on we will demonstrate that this different behavior of the correlations for both cutoff values does not show up in observables.

One needs a second condition to fix both LECs uniquely. The $n d$ doublet scattering length ${ }^{2} a_{n d}$ is known to be correlated with the ${ }^{3} \mathrm{H}$ BE. This correlation is known as the Phillips line [37]. We investigated it in the context of chiral nuclear forces. It turns out that the scattering length depends on $c_{D}$ even if $c_{E}$ is chosen according to the correlation in Fig. 2 with the fixed value for the triton BE. This indicates that the correlation between the doublet scattering length ${ }^{2} a_{n d}$ and the ${ }^{3} \mathrm{H} \mathrm{BE}$ is not exact. In fact, already for conventional $N N$ and $3 N$ forces, there was a slight scatter around an average line correlating the ${ }^{3} \mathrm{H}$ and ${ }^{2} a_{n d}$ values for different nuclear forces $[80,38]$. The Phillips line has recently been rediscovered within pion-less EFT [39-41]. At 

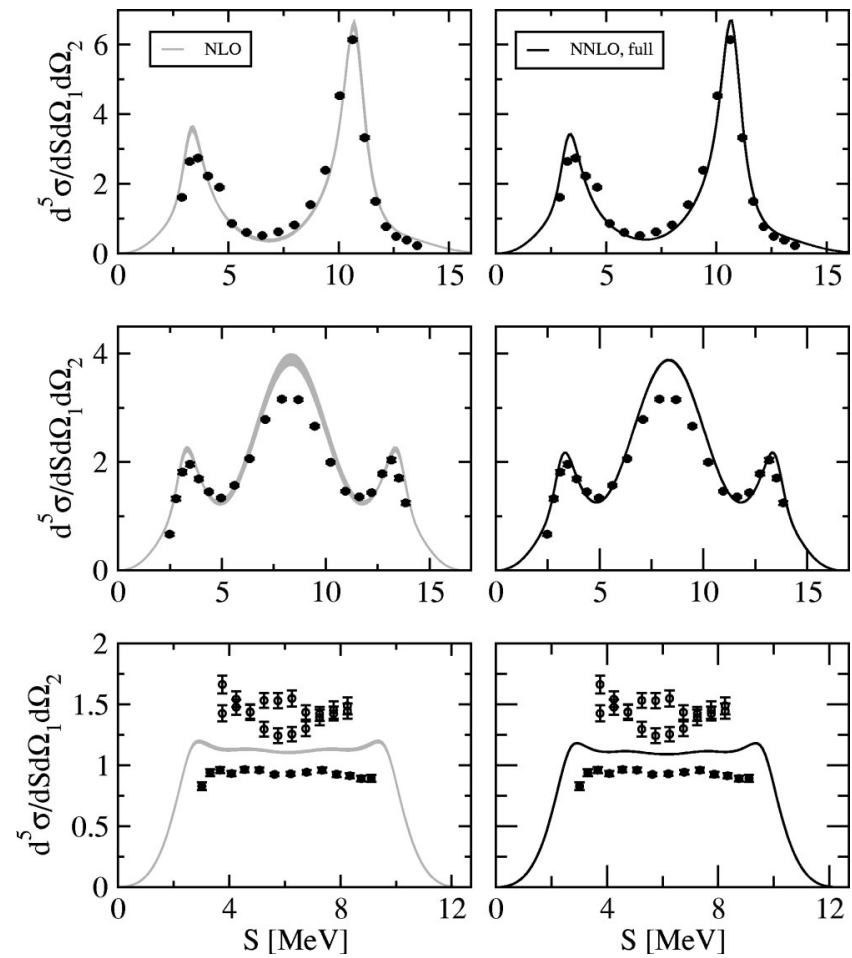

FIG. 8. nd break-up cross section in $\left(\mathrm{mb} \mathrm{MeV}-1 \mathrm{sr}^{-2}\right)$ along the kinematical locus $S$ (in $\mathrm{MeV}$ ) at $13 \mathrm{MeV}$ in comparison to predictions at NLO (light shaded band) and NNLO (dark shaded band) in chiral effective field theory. In the upper row a final state interaction configuration is shown, in the middle one a quasifree scattering configuration (both in comparison to $p d$ data) and in the lower one a space-star configuration (upper data $n d$, lower data $p d$ ). The precise kinematical description can be found in Ref. [25]. $p d$ data are from Ref. [70], nd data from Refs. [71,72].

LO and NLO in the pion-less EFT the $3 \mathrm{NF}$ is given by a single contact term without derivatives and thus depends on just one free parameter. The Phillips line results from variation of this parameter and is in agreement with results based upon phenomenological interactions. Going to higher orders in the low-momentum expansion one encounters contributions to the $3 \mathrm{NF}$ with more derivatives and the exact correlation between ${ }^{2} a_{n d}$ and ${ }^{3} \mathrm{H} \mathrm{BE}$ observed at LO and NLO is broken, see [40] for more details. As discussed above, in the EFT with explicit pions the first nonvanishing $3 \mathrm{NF}$ at NNLO already depends on two free parameters and thus the Phillips line is already broken at this order in the chiral expansion. This allows to determine $c_{D}$ (and at the same time $c_{E}$ ) by a fit to the "experimental" pseudodatum for the doublet scattering length. In Fig. 3 the gray horizontal band indicates the scattering length range in agreement with the experimental error bar. Our theoretical predictions for $\Lambda=500$ and $600 \mathrm{MeV}$ are shown against $c_{D}$. We read off from Figs. 2 and 3 the following values:

$$
\begin{gathered}
c_{D}=3.6, \quad c_{E}=0.37, \quad \Lambda=500 \mathrm{MeV}, \\
c_{D}=1.8, \quad c_{E}=-0.11, \quad \Lambda=600 \mathrm{MeV} .
\end{gathered}
$$
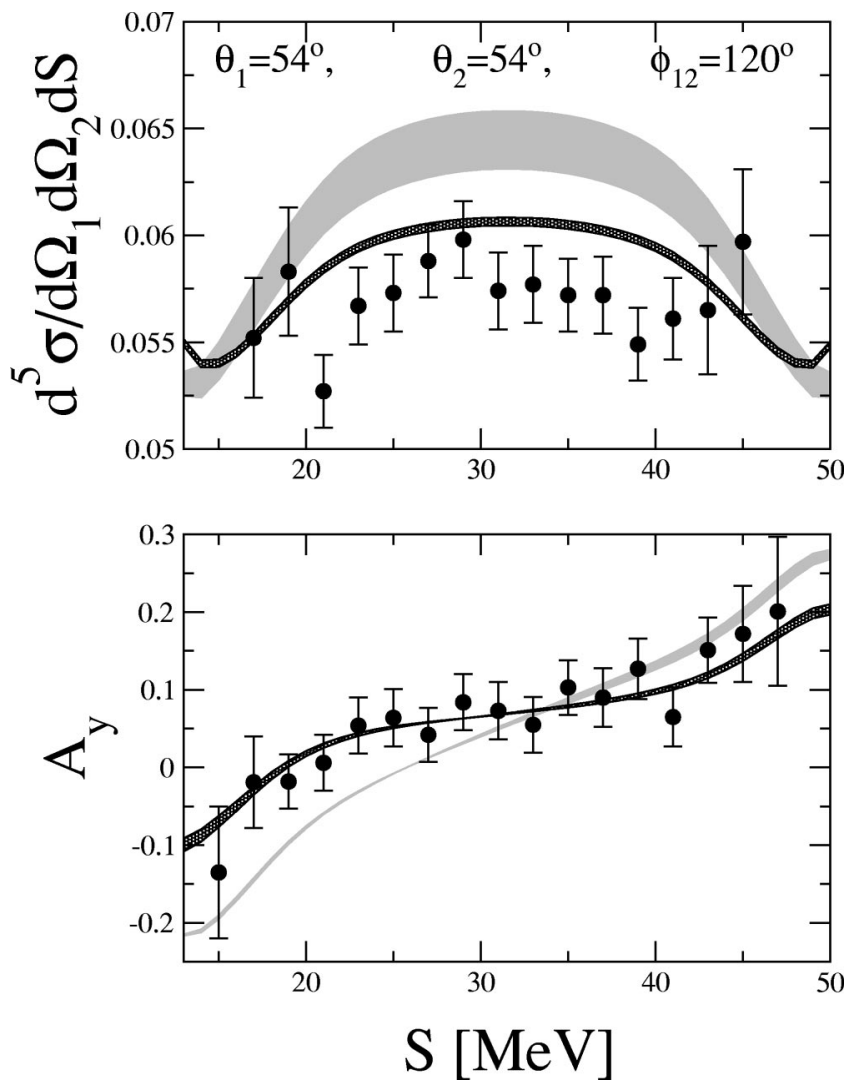

FIG. 9. nd break-up cross section in $\left(\mathrm{mb} \mathrm{MeV}^{-1} \mathrm{sr}^{-2}\right)$ and nucleon analyzing power along the kinematical locus $S$ (in $\mathrm{MeV}$ ) at $65 \mathrm{MeV}$ in comparison to predictions at NLO (light shaded band) and NNLO (dark shaded band) in chiral effective field theory. Symmetric space-star configuration is shown. $p d$ data are from Ref. [73].

Notice that the sign of the determined LEC $c_{D}$ agrees with the one found in [30] from $P$-wave pion production in the proton-proton collisions. Note that for comparing our results with the ones of [30] one should take into account different conventions with respect to $g_{A}$. Also it should be understood that only a qualitative comparison (if at all) for the value of $c_{D}$ can be performed due to different regularization schemes used in our work and in [30]. We are aware of the fact that we can only obtain a first estimate of $c_{D}$ and $c_{E}$. The most important uncertainties are the errors due to the $n p$ force corrections and the experimental error bar of ${ }^{2} a_{n d}$. In principle the errors due to these uncertainties with respect to observables could be estimated performing calculations with several $c_{D}$ and $c_{E}$ combinations consistent with these error bars. In view of upcoming new data for ${ }^{2} a_{n d}[42,43]$ and work on the isospin breaking in our formalism, we postpone such an analysis. In summary we emphasize that the breakdown of the Phillips line correlation enables us to determine the LECs from the $3 N \mathrm{BE}$ and the $n d$ doublet scattering length. The result is a parameter free $3 N$ Hamiltonian. In the next sections we will investigate the results for the $4 N$ bound state and $3 N$ scattering based on this Hamiltonian. 

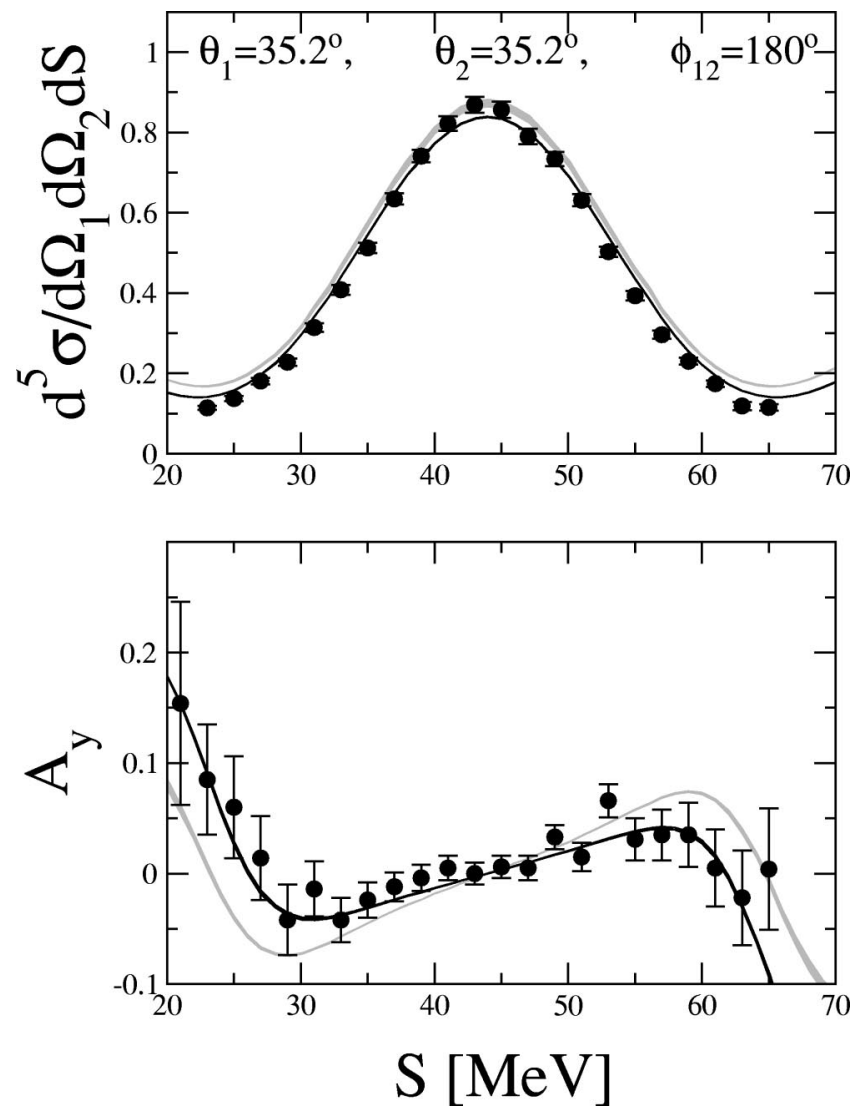

FIG. 10. $n d$ break-up cross section in $\left(\mathrm{mb} \mathrm{MeV}^{-1} \mathrm{sr}^{-2}\right)$ and nucleon analyzing power along the kinematical locus $S$ (in $\mathrm{MeV}$ ) at $65 \mathrm{MeV}$ in comparison to predictions at NLO (light shaded band) and NNLO (dark shaded band) in chiral effective field theory. Symmetric forward star configuration is shown. $p d$ data are from Ref. [73].

\section{PREDICTIONS FOR THREE- AND FOUR-NUCLEON SYSTEMS}

We start with the prediction for the $\alpha$-particle BE. This is based on the solution of Yakubovsky equations [44] as described in $[32,45]$. The results are fully converged and accurate to $2 \mathrm{keV}$ for the $3 N$ and $20 \mathrm{keV}$ for the $4 N$ system. The convergence with respect to partial waves is much faster for the chiral interactions than for the conventional ones. This is a consequence of the momentum cutoffs, which suppress the high momentum components exponentially. The calculations of the binding energy for the chiral interactions are truncated at a two-body total angular momentum in the subsystem of $j_{\max }=6$ for the $3 N$ system. For the $4 N$ system we truncate the partial wave decomposition by the restriction that the sum of all three angular momentum quantum numbers is below $l_{\text {sum }}^{\max }=10$. Calculations for conventional forces require $l_{\text {sum }}^{\max }=14$ (for details see [32]).

Before we comment on our results for the BEs, we need to define a Coulomb and $n p$ corrected $\alpha$-particle BE. Again, based on AV18+Urbana-IX and CD-Bonn+TM99', we calculated BEs for the $\alpha$-particle of $28.5 \mathrm{MeV}$ and $28.4 \mathrm{MeV}$. Replacing the $p p$ and $n n$ forces by $n p$ forces and omitting the Coulomb force, the BEs change to $29.9 \mathrm{MeV}$ and 30.0
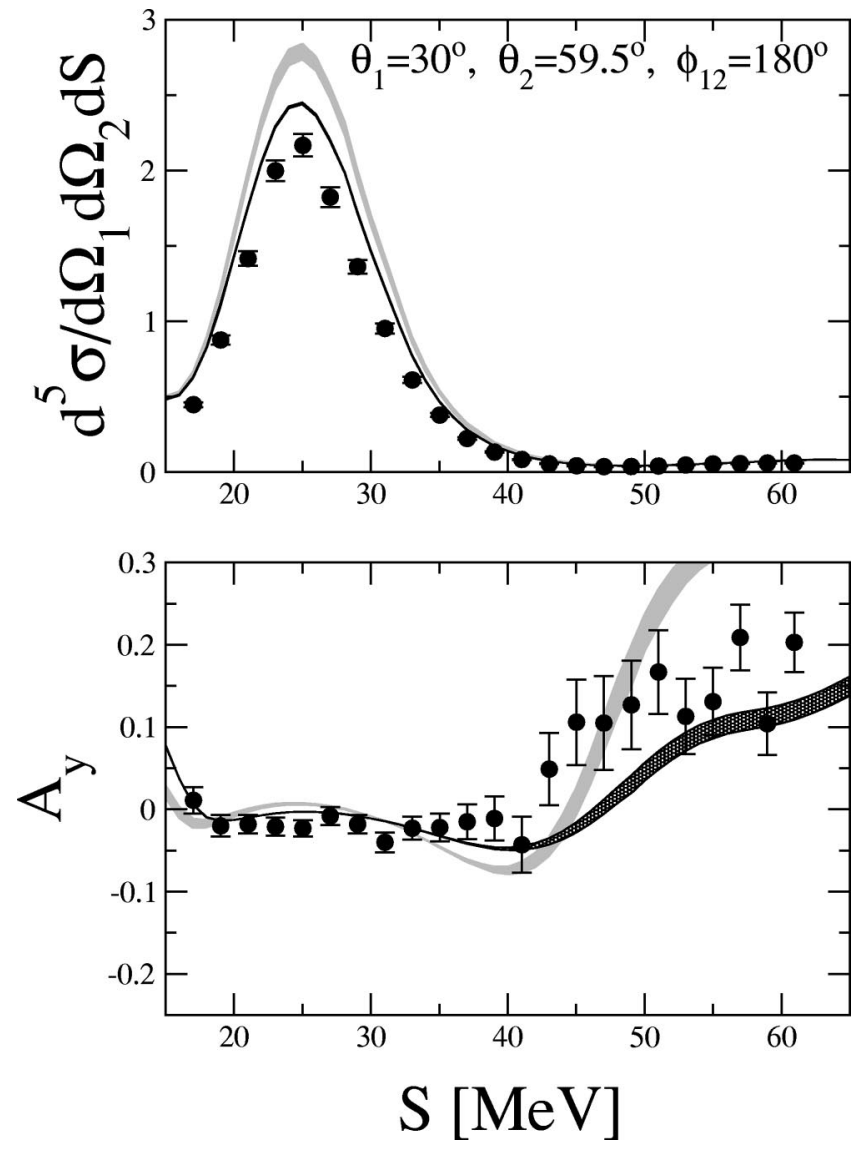

FIG. 11. nd break-up cross section in $\left(\mathrm{mb} \mathrm{MeV}^{-1} \mathrm{sr}^{-2}\right)$ and nucleon analyzing power along the kinematical locus $S$ (in $\mathrm{MeV}$ ) at $65 \mathrm{MeV}$ in comparison to predictions at NLO (light shaded band) and NNLO (dark shaded band) in chiral effective field theory. Quasifree scattering configuration is shown. $p d$ data are from Ref. [73].

$\mathrm{MeV}$. From these results we estimate an average change of the $\mathrm{BE}$ of $1.5 \pm 0.1 \mathrm{MeV}$. The experimental $\alpha$-particle BE is 28.3 MeV. Thus we compare our results for the chiral interaction to an "experimental" pseudo-BE of $29.8 \pm 0.1 \mathrm{MeV}$.

In Table I this value is shown together with the "experimental" pseudo-BE for ${ }^{3} \mathrm{H}$ in comparison to the NLO and NNLO results for ${ }^{3} \mathrm{H}$ and ${ }^{4} \mathrm{He}$. The $\mathrm{BE}$ is in general very sensitive to small changes of the interaction, as it comes out as the difference of the large kinetic and potential energies. As a consequence, we found a rather large dependence of the BEs on the cutoff at NLO [16] ( $\sim 19 \%$ for the $\alpha$-particle). At NNLO the ${ }^{3} \mathrm{H}$ BE agrees with the "experimental" value by construction. Because of the strong correlation of $3 N$ and $4 N$ BEs, known as Tjon-line [46], one can expect a rather small cutoff dependence of the $\alpha$-particle BE, too. This is indeed the case. However, we would like to mention that 3 NFs break this correlation [32] and we observe a $c_{D}$ dependence of the $\alpha$-particle $\mathrm{BE}$ (1 $\mathrm{MeV}$ change in the range $c_{D}$ $=-1.5+1.5$ for $\Lambda=500 \mathrm{MeV})$. We are also very encouraged by the fact that the $\alpha$-particle BE for both cutoffs comes out close to the "experimental" value. Note also that no $4 \mathrm{NF}$ contributes at NNLO. Therefore all predictions for $A>3$ at NNLO are parameter free.

Additionally, we list the expectation values of the differ- 

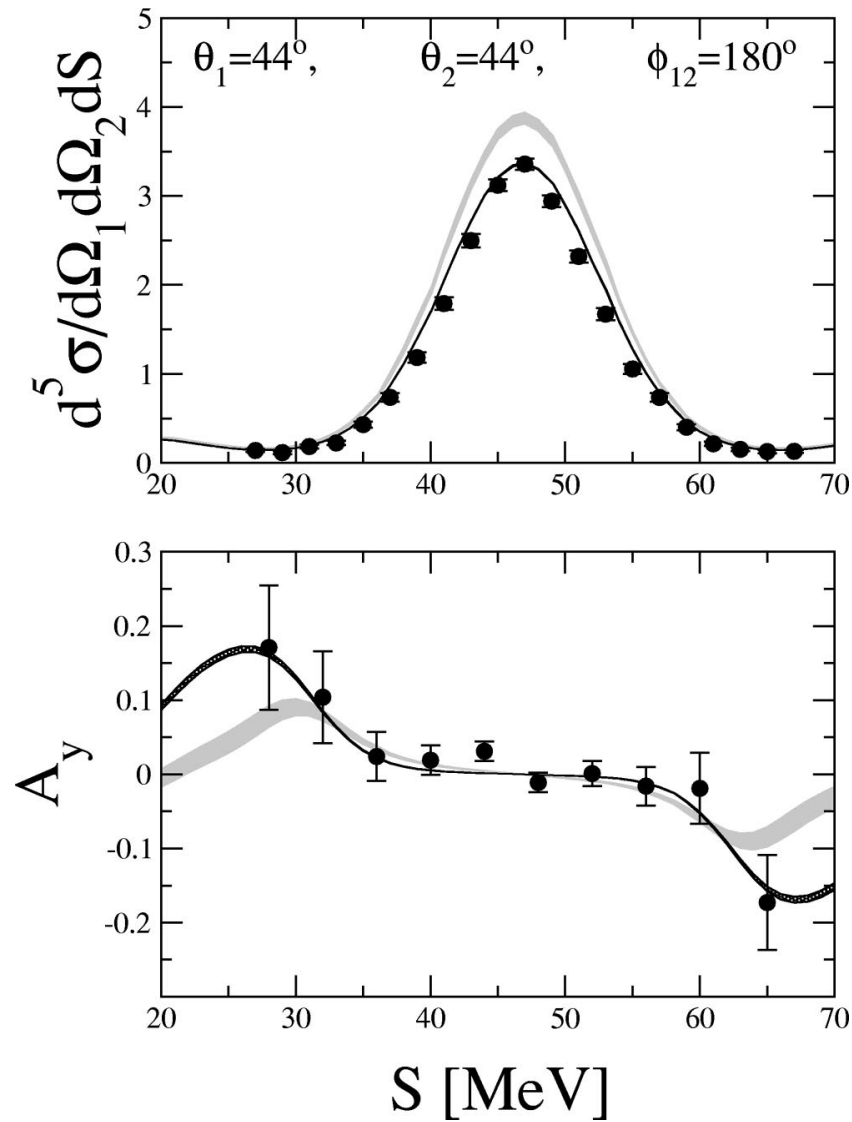

FIG. 12. $n d$ break-up cross section in $\left(\mathrm{mb} \mathrm{MeV}^{-1} \mathrm{sr}^{-2}\right)$ and nucleon analyzing power along the kinematical locus $S$ (in $\mathrm{MeV}$ ) at $65 \mathrm{MeV}$ in comparison to predictions at NLO (light shaded band) and NNLO (dark shaded band) in chiral effective field theory. Quasifree scattering configuration is shown. $p d$ data are from Ref. [73].

ent parts of the Hamiltonian in Table I. It is important to realize that those quantities are not observable. We see that the relative contributions of the $N N$ and $3 \mathrm{NF}$ parts are comparable in the $3 N$ and $4 N$ system. We also observe that the ratio of $N N$ and $3 \mathrm{NFs}$ strongly depends on the cutoff chosen. This is elaborated in more detail in Table II, where the $3 \mathrm{NF}$ expectation value is split in the contribution from the $2 \pi$ exchange ( $c$ terms), $1 \pi$ exchange ( $D$ term) and contact term ( $E$ term). The contributions of $D$ and $E$ term cancel to a large extent in both nuclei and for both cutoffs. The change in sign of the $E$ term changing from $\Lambda=500 \mathrm{MeV}$ to $\Lambda$ $=600 \mathrm{MeV}$ has to be expected, since $c_{E}$ changes its sign, too. More surprising is the change in sign for the $D$ term. This has to be caused by a qualitatively different action of the $D$ term operator on the wave functions for $\Lambda$ $=500 \mathrm{MeV}$ and $\Lambda=600 \mathrm{MeV}$.

We now switch to scattering observables. Most of the $3 \mathrm{~N}$ scattering data have been obtained for the $p d$ system. In the case of scattering the isospin breaking effects in the nuclear force are believed to be of minor importance. We have checked this assumption explicitly for elastic scattering observables using the CD Bonn potential with $n p$ and $n n$ and with only $n p$ forces to evaluate corresponding effects. Only in two cases at $3 \mathrm{MeV}$, namely, for $T_{20}$ (at forward angles
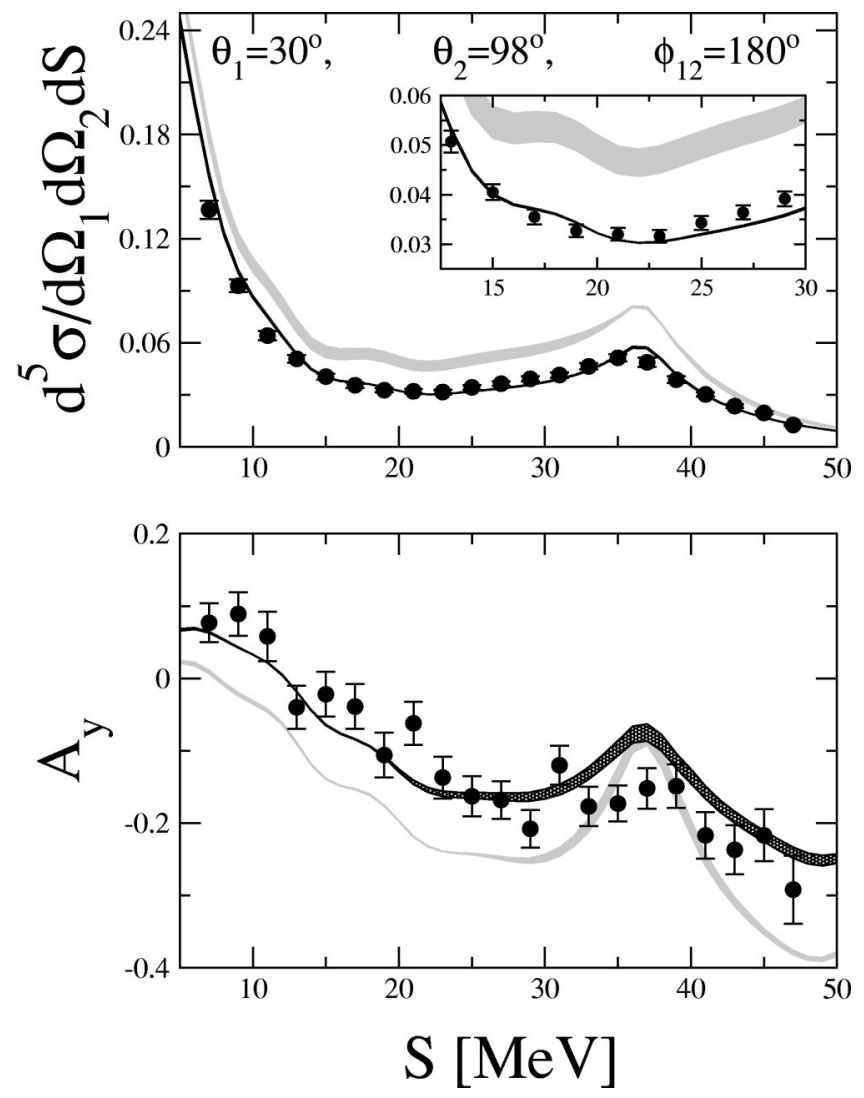

FIG. 13. nd break-up cross section in $\left(\mathrm{mb} \mathrm{MeV}^{-1} \mathrm{sr}^{-2}\right)$ and nucleon analyzing power along the kinematical locus $S$ (in $\mathrm{MeV}$ ) at $65 \mathrm{MeV}$ in comparison to predictions at NLO (light shaded band) and NNLO (dark shaded band) in chiral effective field theory. Collinear configuration is shown. $p d$ data are from Ref. [74].

data are shifted upwards) and for $T_{21}$ (data are shifted downwards at angles below $120^{\circ}$ ), significant effects were found. The $n p$-force corrections are small for all considered elastic scattering observables at $10 \mathrm{MeV}$ and nearly invisible at 65 $\mathrm{MeV}$. Therefore we refrain from correcting data for this effect. In contrast, there are visible Coulomb corrections necessary at these energies. We are not able to take the Coulomb force into account in the $3 \mathrm{~N}$ continuum. For the Coulomb corrections we rely on the work of the Pisa collaboration, who can calculate low energy scattering observables based on the full AV18 interaction including the Coulomb force $[47,48]$. The difference of these full calculations and calculations without Coulomb force serves as our estimate of the Coulomb corrections. In the following, all $p d$ elastic scattering data at $3 \mathrm{MeV}$ and $10 \mathrm{MeV}$ have been corrected by this amount. For $65 \mathrm{MeV}$ we did not correct the data assuming that Coulomb corrections are small except in forward direction. For the break-up we refrained from any corrections because of the lack of reliable theoretical calculations taking the Coulomb force into account.

The $n d$ scattering observables have been studied very intensively using the modern phenomenological interactions $[25,48]$. In general, the description of the data by these models is very good at low energies with a few prominent exceptions. The most famous one entered the literature as $A_{y}$ 

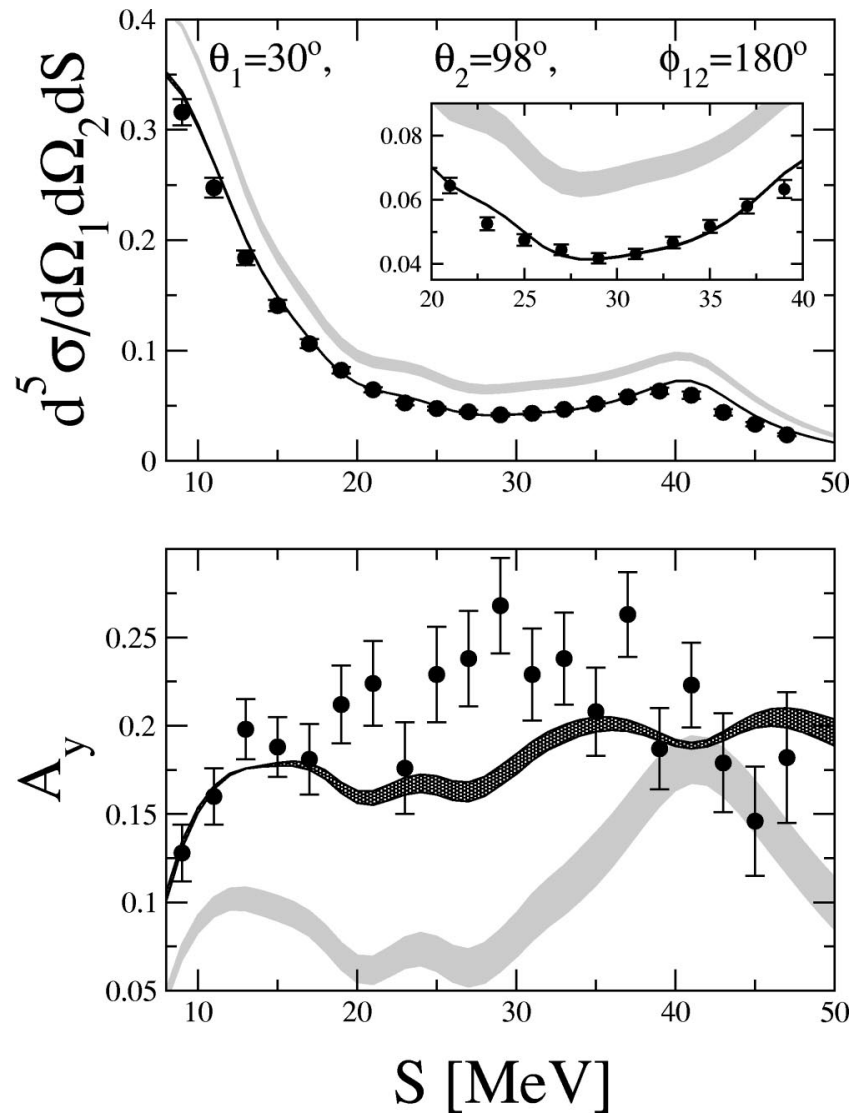

FIG. 14. $n d$ break-up cross section in $\left(\mathrm{mb} \mathrm{MeV}^{-1} \mathrm{sr}^{-2}\right)$ and nucleon analyzing power along the kinematical locus $S$ (in $\mathrm{MeV}$ ) at $65 \mathrm{MeV}$ in comparison to predictions at NLO (light shaded band) and NNLO (dark shaded band) in chiral effective field theory. Collinear configuration is shown. $p d$ data are from Ref. [74].

puzzle $[49,50]$, which is related to the fact that this observable is underpredicted in the maximum by realistic highprecision models of the nucleon interactions. In this paper we do not compare the new results to traditional ones. That has been done in [10] for the NLO and NNLO interactions without $3 \mathrm{NF}$ part. In this case, the NNLO interaction compares quite well to the results [51] based on the highly accurate phenomenological forces. In the following we would like to concentrate on the complete analysis at NNLO.

In Figs. 4, 5, and 6 we show a comparison for few selected elastic scattering observables at 3,10 , and $65 \mathrm{MeV}$, respectively. The left column shows our results for NLO [52] in comparison to the data and the right column the new NNLO results compared to the same data. The bands are given by the cutoff variation in the range from $\Lambda=500$ and $\Lambda=600 \mathrm{MeV}$. They may serve as an estimation of the effects due to neglected higher order terms in the chiral expansion. Since we are not able to vary the cutoff in a much larger range for the reasons explained above, a better estimation of the theoretical uncertainty would be to incorporate the $\mathrm{N}^{3} \mathrm{LO}$ terms and to see effects in observables by varying the corresponding coupling constants in the natural range. We however refrain from doing such a study here.

The differential cross section is presented in the first row of Figs. 4, 5, and 6. Additionally, we give a more detailed
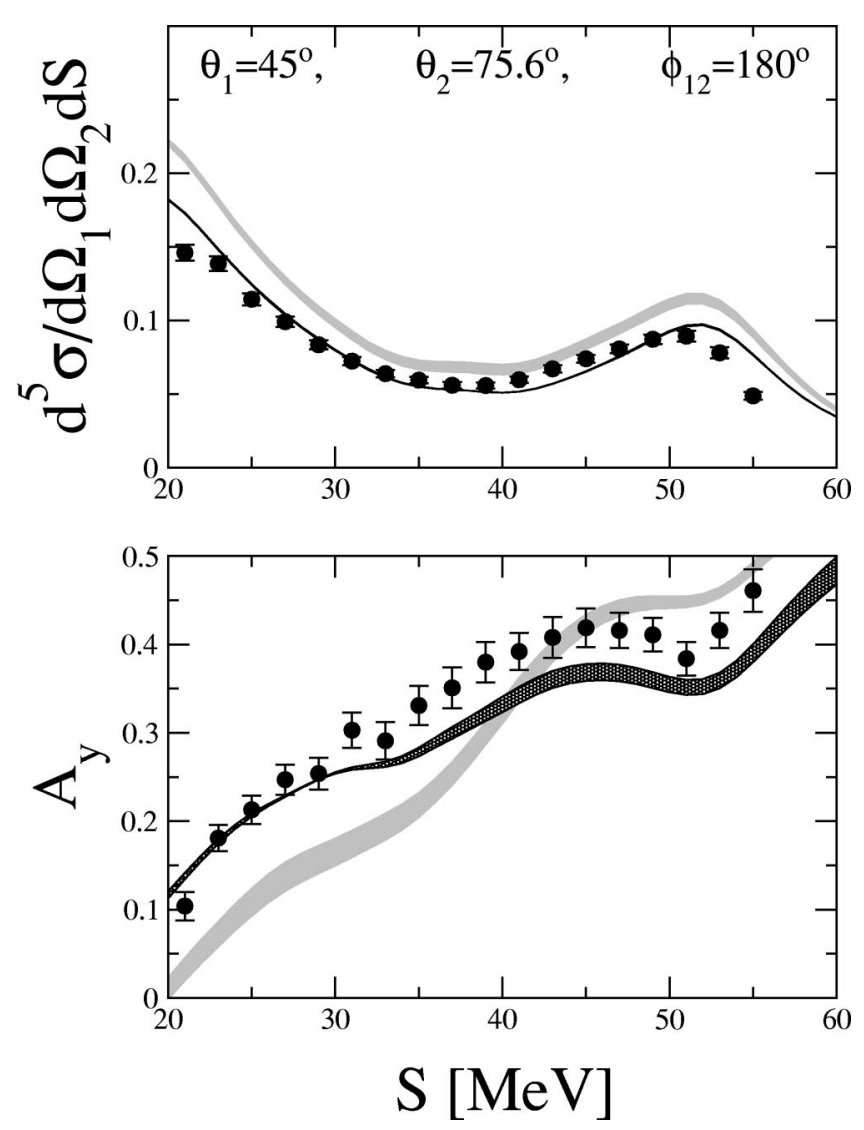

FIG. 15. nd break-up cross section in $\left(\mathrm{mb} \mathrm{MeV}^{-1} \mathrm{sr}^{-2}\right)$ and nucleon analyzing power along the kinematical locus $S$ (in $\mathrm{MeV}$ ) at $65 \mathrm{MeV}$ in comparison to predictions at NLO (light shaded band) and NNLO (dark shaded band) in chiral effective field theory. Collinear configuration is shown. $p d$ data are from Ref. [74].

look at the cross section minima in Fig. 7. At $3 \mathrm{MeV}$ and 10 $\mathrm{MeV}$ we see that NLO and NNLO predictions overlap. The cutoff dependence is already small at NLO and nearly vanishes at NNLO. This strong reduction of the cutoff dependence of this observable at NNLO is expected and can easily be understood. Indeed, at least at low energy the differential cross section is dominated by the $2 N S$ waves. The situation is more interesting at $65 \mathrm{MeV}$. In the minimum of the cross section one observes large differences between the NLO and NNLO results (also to the incomplete NNLO calculation, see [10]). The cutoff dependence of the NLO results is more visible than at lower energies, and is again strongly reduced at NNLO. The NNLO results are in agreement with the data except for forward directions, which are sensitive to the Coulomb force. Note that the improvement at NNLO is not only due to the fact that the NNLO $2 N$ potential leads to a much more accurate description of the data especially at moderate energies [10], but also due to the chiral 3NF. This is demonstrated by the dotted line in the lower panel of Fig. 7, which corresponds to $c_{D}=-3.0$ at $\Lambda=500 \mathrm{MeV}$ (and a $c_{E}$ chosen appropriately to reproduce the $3 N$ binding energy) [81]. For this value of $c_{D}$ the prediction in the minimum is in disagreement with the data. It is gratifying to see that fixing the LEC $c_{D}$ from the scattering data at zero energy we are able to describe the cross section minimum at $65 \mathrm{MeV}$. We consider 

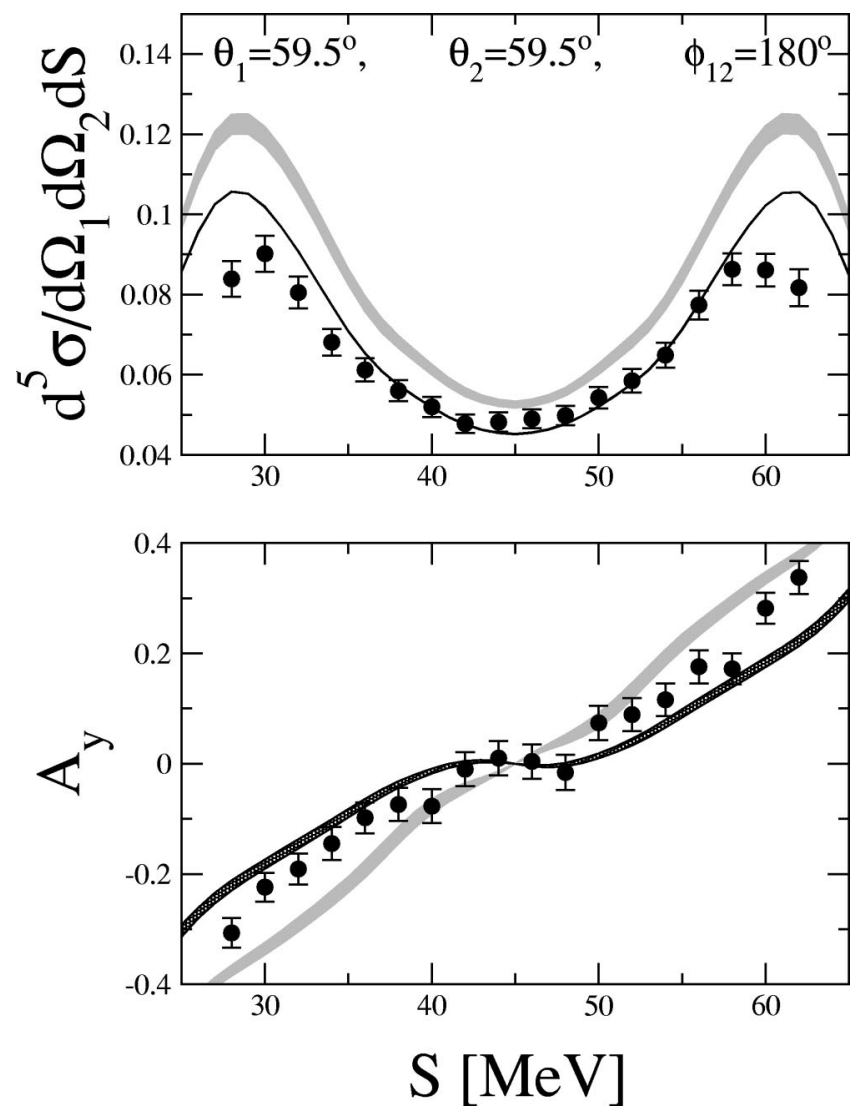

FIG. 16. nd break-up cross section in $\left(\mathrm{mb} \mathrm{MeV}^{-1} \mathrm{sr}^{-2}\right)$ and nucleon analyzing power along the kinematical locus $S$ (in $\mathrm{MeV}$ ) at $65 \mathrm{MeV}$ in comparison to predictions at NLO (light shaded band) and NNLO (dark shaded band) in chiral effective field theory. Collinear configuration is shown. $p d$ data are from Ref. [74].

this to be an important indication of consistency in the determination of the LECs $c_{D}$ and $c_{E}$.

As already pointed out before, the most problematic observable of $n d$ elastic scattering is $A_{y}$, which is shown in the second row of Figs. 4, 5, and 6. First of all we would like to stress that vector and tensor analyzing powers are defined as differences of polarized cross sections and are rather small at low energies, so that larger theoretical errors for these observables have to be expected. At energies 3 and $10 \mathrm{MeV}$ we see visible deviations of our predictions for $A_{y}$ from the data for both NLO and NNLO. It is well known [53] that this observable is extremely sensitive to the ${ }^{3} P$-wave phase shifts in the $N N$ system. Although at NLO chiral predictions at $3 \mathrm{MeV}$ seem to be in agreement with the data, this cannot be considered as a final result in chiral EFT. Indeed, the ${ }^{3} P$-wave phase shifts in the $N N$ system are only described at low energies with an accuracy of about 5\% [10], which indicates that large corrections to $n d A_{y}$ at higher orders in the chiral expansion are possible. At NNLO the ${ }^{3} P$-wave phase shifts come out with a significantly smaller error of about $2 \%$ (at $T_{\text {lab }}=10 \mathrm{MeV}$ ) and we found in [10] that $A_{y}$ is underpredicted if one only uses the $2 \mathrm{~N}$ forces, just as in the case of high-precision potential models. As one sees from Figs. 4 and 5, we do not solve the $A_{y}$ puzzle performing the complete NNLO analysis and including the 3NF. It is important
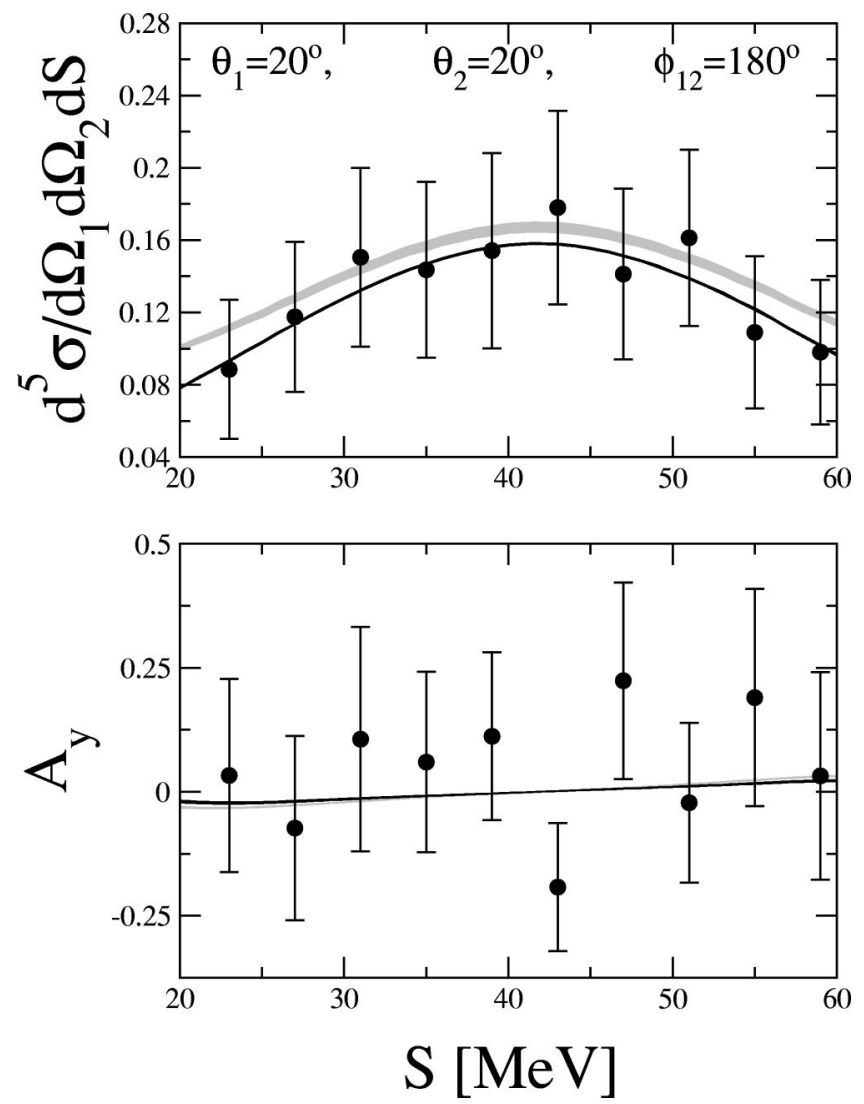

FIG. 17. $n d$ break-up cross section in $\left(\mathrm{mb} \mathrm{MeV}^{-1} \mathrm{sr}^{-2}\right)$ and nucleon analyzing power along the kinematical locus $S$ (in $\mathrm{MeV}$ ) at $65 \mathrm{MeV}$ in comparison to predictions at NLO (light shaded band) and NNLO (dark shaded band) in chiral effective field theory. Unspecific configuration is shown. $p d$ data are from Ref. [75].

to stress that in principle, one could try to solve this puzzle by the NNLO chiral 3NF. Indeed, instead of fixing the unknown LECs $c_{D}$ and $c_{E}$ to the triton binding energy and the doublet scattering length, one could think about requiring a good description of $A_{y}$ at, say, $3 \mathrm{MeV}$ as being one of the two conditions needed. We found, however, that $A_{y}$ is not very sensitive to the choice of the LECs $c_{D}$ and $c_{E}$, if the two are adjusted to reproduce the triton binding energy. We were not able to find values of these coefficients in the natural range, which would simultaneously describe $A_{y}$ at $3 \mathrm{MeV}$ and the triton $\mathrm{BE}$ [82]. In particular, it turns out that negative values of $c_{D}\left(c_{E}\right.$ being fixed according to Fig. 2) lead to a slight improvement for $A_{y}$. For example, at $E_{\text {lab }}=3 \mathrm{MeV}$ one observes a shift in the maximum in $A_{y}$ by $+2.6 \%$ for $c_{D}=-3$ and $\Lambda=500 \mathrm{MeV}$ compared to the result obtained without $3 \mathrm{NF}$ of the $D$ and $E$ types, and which still deviates from the data by more than $10 \%$. Taking larger negative values of $c_{D}$ does not lead to further significant improvement. Note also that such negative values of the $\operatorname{LEC} c_{D}$ are inconsistent with the data for the nd doublet scattering length, $\alpha$-particle binding energy as well as for the differential cross section at moderate energies. This significant disagreement with the data for $A_{y}$ at low energies may be an indication that higher order effects are still important for this observable. On the other hand, it can be also a hint that the 

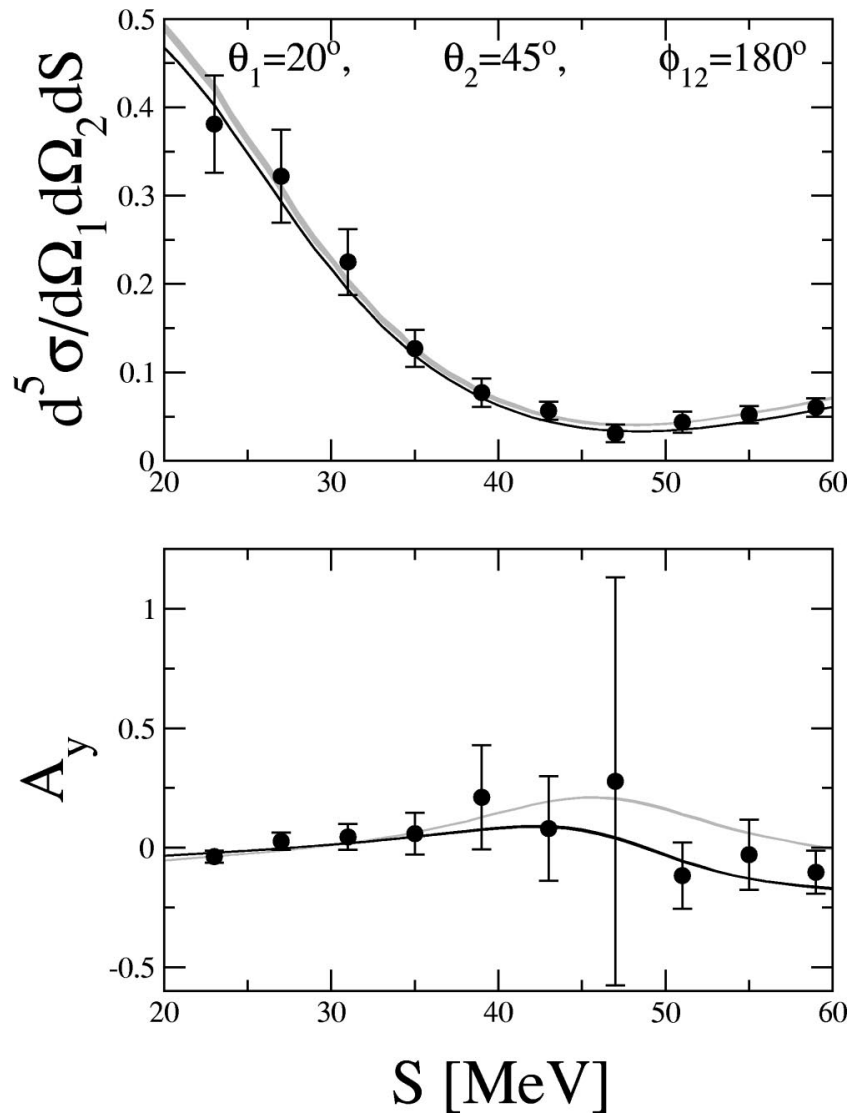

FIG. 18. nd break-up cross section in $\left(\mathrm{mb} \mathrm{MeV}^{-1} \mathrm{sr}^{-2}\right)$ and nucleon analyzing power along the kinematical locus $S$ (in $\mathrm{MeV}$ ) at $65 \mathrm{MeV}$ in comparison to predictions at NLO (light shaded band) and NNLO (dark shaded band) in chiral effective field theory. Unspecific configuration is shown. $p d$ data are from Ref. [75].

$A_{y}$ puzzle is related to an insufficient knowledge of the lowenergy ${ }^{3} P_{j} N N$ phases [54]. It will be interesting, as a next step, to include $1 / m_{N}$ corrections to the interactions and to study their effect especially on $A_{y}$. At $65 \mathrm{MeV}$ the decription of $A_{y}$ is much better, which is in agreement with results based upon the phenomenological nuclear forces.

The lower three rows of Figs. 4, 5, and 6 show the tensor analyzing powers $T_{20}, T_{21}$, and $T_{22}$. At $3 \mathrm{MeV}$ and $10 \mathrm{MeV}$, we find in general that the NNLO predictions stay within the NLO band. The cutoff dependence clearly shrinks, which is a good indication of convergence of the chiral expansion. Altogether the agreement with the data is good except for $T_{20}$ and $T_{21}$ at $10 \mathrm{MeV}$ [83]. Notice that similar results have been reported in [55] based upon the combination of the AV18 $2 \mathrm{~N}$ and the Urbana IX $3 \mathrm{~N}$ forces, where these observables have been calculated in the $p d$ system and the Coulomb force has been taken into account. At $65 \mathrm{MeV}$, the situation is comparable to the one for the cross section. While the NLO predictions at this energy deviate significantly from the data, the NNLO results are in a much better agreement. Unfortunately, the quality of the data does not allow to draw more precise conclusions and especially here new high-precision data are needed.

Let us now switch to break-up observables. We performed calculations at two energies, 13 and $65 \mathrm{MeV}$, where a lot of
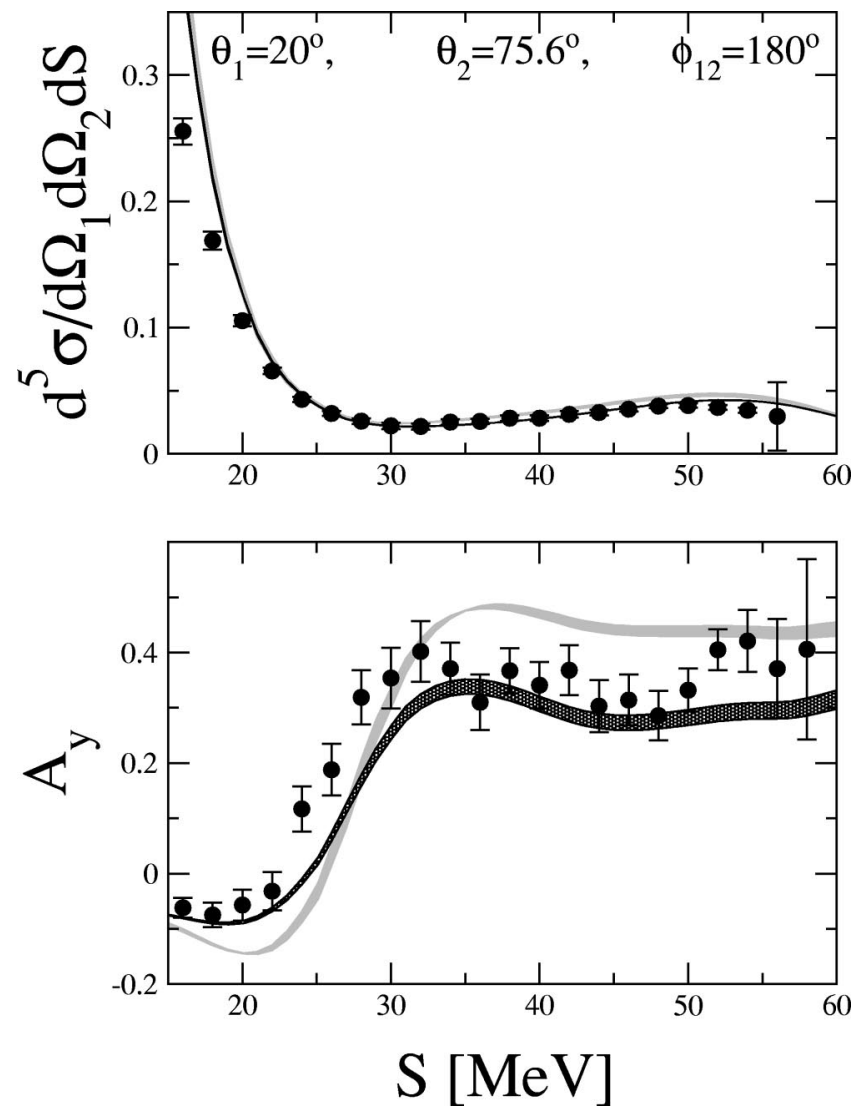

FIG. 19. nd break-up cross section in $\left(\mathrm{mb} \mathrm{MeV}^{-1} \mathrm{sr}^{-2}\right)$ and nucleon analyzing power along the kinematical locus $S$ (in $\mathrm{MeV}$ ) at $65 \mathrm{MeV}$ in comparison to predictions at NLO (light shaded band) and NNLO (dark shaded band) in chiral effective field theory. Unspecific configuration is shown. $p d$ data are from Ref. [75].

pd data exist. As already pointed out above, there are no reliable Coulomb corrections available for the break up. Therefore we show the noncorrected $p d$ data in comparison to our $n d$ calculations. Note that for the space star configuration at $13 \mathrm{MeV}$ presented in Fig. 8, it is shown that the $n d$ and $p d$ cross section data strongly deviate indicating that Coulomb effects can become important at least in some configurations. Presumably, the Coulomb corrections are smaller at $65 \mathrm{MeV}$.

At $13 \mathrm{MeV}$ we demonstrate in Fig. 8 chiral predictions for the cross section in the often investigated final-state interaction peak, quasifree scattering and space-star configurations, which have also been considered in the NLO analysis [16]. For a general discussion on various break-up observables and configurations the reader is referred to Ref. [25]. As demonstrated in Fig. 8, the NLO and NNLO results essentially agree at $13 \mathrm{MeV}$. They describe the configuration dominated by FSI peaks quite well (for a more detailed comparison the angular openings of the detectors have to be taken into account, see [25]). The present theory for the break-up configuration including a QFS geometry fails in the central maximum. This might be due to Coulomb force effects. The third configuration, the so-called space-star, is one of the long standing puzzles of $3 N$ scattering [56-60]. Similar to results with phenomenological interactions, we 
even fail to describe the $n d$ data. $p d$ and $n d$ data are quite different and it remains open whether Coulomb corrected data would fall on the theory. We observe [25] the tendency that with conventional $N N$ forces theory is already rather close to the $p d$ data at $19 \mathrm{MeV}$ and even closer at $65 \mathrm{MeV}$. This suggests that presumably the discrepancy to $p d$ data is due to Coulomb force effects.

At $65 \mathrm{MeV}$ we decided to present the results for the same configurations as the ones studied recently in the context of phenomenological nuclear forces $[56,57]$ in order to enable a direct comparison between these two different approaches. We follow the lines of $[56,57]$ and include, in addition to the cross section, also $A_{y}$. The situation at $65 \mathrm{MeV}$ seems in general to be very promising as documented in Figs. 9-20. The improvements in the description of the data in going from NLO to NNLO are quite impressive. It is interesting that sometimes in case of $A_{y}$ the band width at NNLO is still relatively large, which indicates that this observable might get significant corrections at higher orders. In Figs. 11 and 16 we fail to describe the cross section in part of the $S$-range. The reason is not known to us. These observables also change visibly, when going from NLO to NNLO. Here we cannot claim that convergence with respect to the chiral expansion is reached at NNLO. For one of the configurations (see Fig. 14), the step for $A_{y}$ going from NLO to NNLO is dramatic and better data would be very welcome. Finally we point to two more cases in Figs. 13 and 14, where the band width in the cross section shrinks nicely going to NNLO and where the agreement with the data is quite good.

In view of the quite good description of the $\mathrm{Nd}$ elastic and break up data at $65 \mathrm{MeV}$ at NNLO and of the good description of the $N N$ data up to $200 \mathrm{MeV}$, we are optimistic and expect to be able to describe the data at NNLO in the energy regime towards $100 \mathrm{MeV}$. From investigations based on phenomenological interactions [51,56], we expect that there $3 \mathrm{NF}$ effects become clearly visible at these higher energies. In addition, observables at these energies will probably be more sensitive to the structure of the $3 N$ interaction. For example, the sensitivity of the cross section minimum to the value of $c_{D}$ observed at $65 \mathrm{MeV}$ is expected to be magnified at higher energies. Therefore we call for more data at intermediate energies, which could be compared to predictions of chiral EFT. Notice also that the existence of such high-quality data will be of a crucial importance for higher order calculations, where more parameters in the $3 \mathrm{NF}$ will have to be fixed from the data and a better accuracy in the theory will be reached.

\section{SUMMARY AND CONCLUSIONS}

In summary we applied for the first time the complete chiral EFT interaction at NNLO to the $3 N$ and $4 N$ bound states and to $3 N$ scattering. We reexamined the $3 \mathrm{NF}$ of the chiral interaction at NNLO and used antisymmetrization to eliminate all parameters except two. We showed that these two parameters can be determined from the ${ }^{3} \mathrm{H} \mathrm{BE}$ and the ${ }^{2} a_{n d}$ scattering length. For the time being the accuracy of the scattering length is not sufficient to perform a very precise determination of these two parameters. However, the favorable description of $n d$ scattering data indicates that the val-
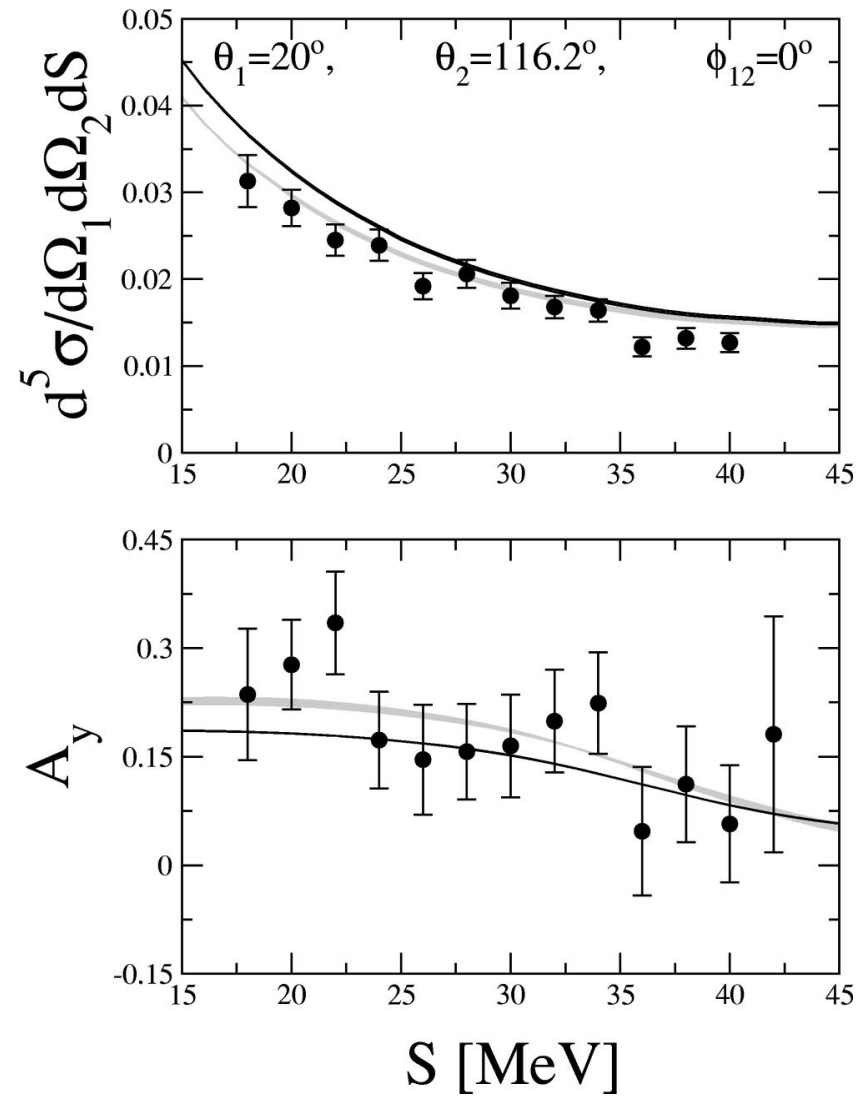

FIG. 20. $n d$ break-up cross section in $\left(\mathrm{mb} \mathrm{MeV}^{-1} \mathrm{sr}^{-2}\right)$ and nucleon analyzing power along the kinematical locus $S$ (in $\mathrm{MeV}$ ) at $65 \mathrm{MeV}$ in comparison to predictions at NLO (light shaded band) and NNLO (dark shaded band) in chiral effective field theory. Unspecific configuration is shown. $p d$ data are from Ref. [75].

ues chosen in this work are in a reasonable range.

We showed that the obtained parameter-free Hamiltonian leads to a good description of the $\alpha$-particle BE. The theory thus seems to be applicable to this densely bound system. It will be interesting to apply the $3 \mathrm{~N}$ Hamiltonian to light nuclei, e.g., using the no-core shell model technique $[61,62]$.

Overall we observe a good description of the data at NNLO. Most of the low energy elastic scattering data (at 3 and $10 \mathrm{MeV}$ ) are described at both orders NLO and NNLO showing convergence of the chiral expansion and agreement with the data. $A_{y}$ turns out to be a problematic observable as there is still no agreement with the data and the predictions for NLO and NNLO disagree. Whether this will be cured by $1 / m$ corrections has to be studied in a forthcoming paper.

At $65 \mathrm{MeV}$ the situation is also very promising. In general, we observe that the NNLO predictions move towards or onto the data, while the NLO results deviate significantly from the data. In Ref. [10] we found that the NNLO interaction can describe the $N N$ phase shifts up to energies of 200 $\mathrm{MeV}$ neutron lab energy. Here we see that the extension of the energy range going to NNLO for the two-body system is continued in the few-body systems.

This study was based on the systematic expansion of the nuclear force according to chiral perturbation theory applied to the $N N$ potential. We emphasize that the favorable agree- 
ment with the data, the stability of our predictions when going from NLO to NNLO, observed in most cases, as well as the decreased cutoff dependence of the NNLO results indicate consistency of our calculations. New nd data in the energy range between $65 \mathrm{MeV}$ and $100 \mathrm{MeV}$ are highly welcome and would allow to draw quantitative conclusions on the range of validity of the NNLO approximation as well as to probe the spin structure of the leading 3NF. Such data would also be of a crucial importance for extending the analysis to higher orders.

In the next steps, we have to take into account the isospin breaking of the nuclear force. Together with upcoming new data for the doublet $n d$ scattering length a much more accurate determination of the $3 \mathrm{NF}$ parameters will then be possible.

\section{ACKNOWLEDGMENTS}

We are very thankful to Alejandro Kievsky for supplying the $p d$ scattering observables based on AV18+Urbana IX and to Jacek Golak for checking the partial wave decomposition of the $3 \mathrm{NF}$ and for the help in numerical problems. E.E. and A.N. would like to thank the hospitality of the Science Center in Benasque, Spain, where a large part of this paper has been written. This work has been partially supported by the Deutsche Forschungsgemeinschaft (E.E.), the U.S. National Science Foundation under Grant No. PHY0070858 (A.N.) and the Polish Committee for Scientific Research under Grant No. 2P03B02818 (H.W.). The numerical calculations have been performed on the Cray T3E and Cray SV1 of the NIC, Jülich, Germany.

\section{APPENDIX: PARTIAL WAVE DECOMPOSITION OF THE CHIRAL 3NF}

Here, we give the explicit formula for the partial wave decomposition of the chiral $3 \mathrm{NF}$. Since the partial wave decomposition of the TPE $3 \mathrm{NF}$ is already discussed, e.g., in [28], we only concentrate here on the remaining contributions to the NNLO $3 \mathrm{NF}$ due to the OPE and contact term in Eq. (10). In [17] the corresponding partial wave decompositions were more involved than what is needed here since the contact terms were expanded by heavy meson propagators. For general details on the partial wave decomposition in the $3 N$ system the reader is referred to Ref. [23]. As already pointed out before, we usually decompose $3 \mathrm{NFs}$ into three parts according to Eq. (14). In the following we give expressions for one such part $V_{3 \mathrm{NF}}^{(i)}$. For the OPE term we find

$$
\begin{aligned}
{ }_{i}\left\langle p q \alpha\left|V_{3 \mathrm{NF}, \mathrm{OPE}}^{(i)}\right| p^{\prime} q^{\prime} \alpha^{\prime}\right\rangle_{i}= & -\frac{9 D g_{A}}{4 F_{\pi}^{2}}(4 \pi)^{2} \delta_{J J^{\prime}} \delta_{M M^{\prime}} \delta_{T T^{\prime}} \delta_{M_{T} M_{T}^{\prime}} \delta_{l 0} \delta_{l^{\prime} 0} \delta_{s j} \delta_{s^{\prime} j^{\prime}}\left[1+(-1)^{s+s^{\prime}+t+t^{\prime}}\right] \\
& \times \sqrt{\hat{s}^{\prime} \hat{j} \hat{I} \hat{I}^{\prime} \hat{t} \hat{t}^{\prime}}(-1)^{j+J+s-I+T+\frac{1}{2}}\left\{\begin{array}{ccc}
\frac{1}{2} & t & T \\
t^{\prime} & \frac{1}{2} & 1
\end{array}\right\}\left\{\begin{array}{ccc}
1 & \frac{1}{2} & \frac{1}{2} \\
\frac{1}{2} & t & t^{\prime}
\end{array}\right\}\left\{\begin{array}{lll}
I & j & J \\
j^{\prime} & I^{\prime} & 1
\end{array}\right\} \\
& \times\left\{\begin{array}{ccc}
1 & \frac{1}{2} & \frac{1}{2} \\
\frac{1}{2} & s & s^{\prime}
\end{array}\right\} \sum_{k_{1}=0,2} \sqrt{\hat{k}_{1}}\left(11 k_{1}, 000\right) \sqrt{\left(2 k_{1}+1\right) !}\left\{\begin{array}{ccc}
1 & k_{1} & 1 \\
\frac{1}{2} & \lambda^{\prime} & I^{\prime} \\
1 & \\
\frac{1}{2} & \lambda & I
\end{array}\right\} \\
& \times \sum_{l_{1}+l_{2}=k_{1}} q^{\prime l_{1}} q^{l_{2}} \frac{1}{\sqrt{\left(2 l_{1}\right) !\left(2 l_{2}\right) !}} \sum_{k} \hat{k} g_{k k_{1}}\left\{\begin{array}{lll}
\lambda & \lambda^{\prime} & k_{1} \\
l_{1} & l_{2} & k
\end{array}\right\}\left(k l_{1} \lambda^{\prime}, 000\right)\left(k l_{2} \lambda, 000\right),
\end{aligned}
$$

where

$$
g_{k k_{1}}=\int_{-1}^{1} d x P_{k}(x) \frac{Q^{2}}{Q^{k_{1}}\left(Q^{2}+M_{\pi}^{2}\right)}
$$

Here $Q \equiv \sqrt{q^{2}+q^{\prime 2}-2 q q^{\prime} x}$ and $P_{k}(x)$ is a Legendre polynomial. Further, $\vec{p}$ and $\vec{q}\left(\vec{p}^{\prime}\right.$ and $\left.\vec{q}^{\prime}\right)$ are relative initial (final) Jacobi momenta in the pair $j k, j, k \neq i$, and of the nucleon $i$ with respect to the pair $j k$, respectively. $l$ and $\lambda\left(l^{\prime}\right.$ and $\lambda^{\prime}$ ) denote the initial (final) relative orbital angular momenta within the pair $j k, j, k \neq i$, and between the nucleon $i$ with respect to the pair $j k$. The initial (final) spin of the subsystem $j k, j, k \neq i$, is denoted by $s\left(s^{\prime}\right)$. In addition, $l$ and $s\left(l^{\prime}\right.$ and $\left.s^{\prime}\right)$ are coupled to the total subsystem angular momentum $j\left(j^{\prime}\right)$, and $\lambda\left(\lambda^{\prime}\right)$ and $s_{i}=\frac{1}{2}$ to the total spectator angular momentum $I\left(I^{\prime}\right)$, which finally combine to $J\left(J^{\prime}\right)$ accompanied by $M\left(M^{\prime}\right)$. The total isospin quantum num- 
bers $T M_{T}\left(T^{\prime} M_{T}^{\prime}\right)$ are constructed analogously: $\left|\left(t \frac{1}{2}\right) T M_{T}\right\rangle\left(\left|\left(t^{\prime} \frac{1}{2}\right) T^{\prime} M_{T}{ }^{\prime}\right\rangle\right)$. We also introduced a convenient abbreviation

$$
\hat{l} \equiv 2 l+1 .
$$

For the contact term in the second line of Eq. (10) we find

$$
\begin{aligned}
{ }_{i}\langle p q \alpha| & \left.\left|V_{3 \mathrm{NF}, \text { cont }}^{(i)}\right| p^{\prime} q^{\prime} \alpha^{\prime}\right\rangle_{i} \\
= & 6 E(4 \pi)^{2} \delta_{J J^{\prime}} \delta_{M M^{\prime}} \delta_{T T^{\prime}} \delta_{M_{T} M_{T}^{\prime}} \delta_{l 0} \delta_{\lambda 0} \delta_{l^{\prime} 0} \delta_{\lambda^{\prime} 0} \delta_{s j} \\
& \times \delta_{s^{\prime} j^{\prime}} \delta_{I \frac{1}{2}}^{1} \delta_{I^{\prime}} \frac{1}{2} \delta_{t t^{\prime}} \delta_{s s^{\prime}}(-1)^{t+1}\left\{\begin{array}{ccc}
\frac{1}{2} & \frac{1}{2} & t \\
\frac{1}{2} & \frac{1}{2} & 1
\end{array}\right\} .
\end{aligned}
$$

[1] S. Weinberg, Phys. Lett. B 251, 288 (1990).

[2] S. Weinberg, Nucl. Phys. B363, 3 (1991).

[3] C. Ordóñez, L. Ray, and U. van Kolck, Phys. Rev. C 53, 2086 (1996).

[4] I. Tamm, J. Phys. (Moscow) 9, 449 (1945).

[5] S.M. Dancoff, Phys. Rev. 78, 382 (1950).

[6] J.L. Friar, Phys. Rev. C 60, 034002 (1999).

[7] E. Epelbaum, W. Glöckle, and Ulf-G. Meißner, Nucl. Phys. A637, 107 (1998).

[8] S. Okubo, Prog. Theor. Phys. 12, 603 (1954).

[9] E. Epelbaum, W. Glöckle, and Ulf-G. Meißner, Nucl. Phys. A671, 295 (2000).

[10] E. Epelbaum, A. Nogga, W. Glöckle, H. Kamada, Ulf.-G. Meißner, and H. Witała, nucl-th/0201064; Eur. Phys. J. A (to be published).

[11] V.G.J. Stoks, R.A.M. Klomp, C.P.F. Terheggen, and J.J. de Swart, Phys. Rev. C 49, 2950 (1994).

[12] U. van Kolck, Phys. Rev. C 49, 2932 (1994).

[13] S.N. Yang and W. Glöckle, Phys. Rev. C 33, 1774 (1986).

[14] J. Gasser and A. Zepeda, Nucl. Phys. B174, 445 (1980).

[15] J.A. Eden and M.F. Gari, Phys. Rev. C 53, 1510 (1996).

[16] E. Epelbaum, H. Kamada, A. Nogga, H. Witała, W. Glöckle, and Ulf-G. Meißner, Phys. Rev. Lett. 86, 4787 (2001).

[17] D. Hüber, J.L. Friar, A. Nogga, H. Witała, and U. van Kolck, Few-Body Syst. 30, 95 (2001)

[18] V. Bernard, N. Kaiser, and Ulf-G. Meißner, Int. J. Mod. Phys. E 4, 193 (1995).

[19] J.L. Friar, D. Hüber, and U. van Kolck, Phys. Rev. C 59, 53 (1999).

[20] P.F. Bedaque, H.-W. Hammer, and U. van Kolck, Nucl. Phys. A676, 357 (2000).

[21] J.L. Friar, Few-Body Syst. 22, 161 (1997).

[22] E. Epelbaum, Ulf.-G. Meißner, W. Glöckle, and Ch. Elster, Phys. Rev. C 65, 044001 (2002).

[23] W. Glöckle, The Quantum Mechanical Few-Body Problem (Springer-Verlag, Berlin, 1983).

[24] A. Nogga, D. Hüber, H. Kamada, and W. Glöckle, Phys. Lett. B 409, 19 (1997).

[25] W. Glöckle, H. Witała, D. Hüber, H. Kamada, and J. Golak, Phys. Rep. 274, 107 (1996).

[26] D. Hüber, H. Kamada, H. Witała, and W. Glöckle, Acta Phys. Pol. B 28, 1677 (1997).

[27] H. Witała, Th. Cornelius, and W. Glöckle, Few-Body Syst. 3, 123 (1988).

[28] D. Hüber, H. Witała, A. Nogga, W. Glöckle, and H. Kamada, Few-Body Syst. 22, 107 (1997).

[29] G.P. Lepage, nucl-th/9706029.
[30] C. Hanhart, U. van Kolck, and G.A. Miller, Phys. Rev. Lett. 85, 2905 (2000).

[31] M. Walzl, Ulf-G. Meißner, and E. Epelbaum, Nucl. Phys. A693, 663 (2001).

[32] A. Nogga, H. Kamada, W. Glöckle, and B.R. Barrett, Phys. Rev. C 65, 054003 (2002).

[33] R.B. Wiringa, V.G.J. Stoks, and R. Schiavilla, Phys. Rev. C 51, 38 (1995).

[34] B.S. Pudliner, V.R. Pandharipande, J. Carlson, S.C. Pieper, and R.B. Wiringa, Phys. Rev. C 56, 1720 (1997).

[35] R. Machleidt, Phys. Rev. C 63, 024001 (2001).

[36] S.A. Coon and H.K. Han, Few-Body Syst. 30, 131 (2001).

[37] A.C. Phillips, Nucl. Phys. A107, 209 (1968).

[38] J.L. Friar, Few-Body Syst., Suppl. 1, 94 (1986).

[39] P.F. Bedaque, H.-W. Hammer, and U. van Kolck, Nucl. Phys. A646, 444 (1999).

[40] P.F. Bedaque and U. van Kolck, Annu. Rev. Nucl. Part. Sci. 52, 339 (2002).

[41] P.F. Bedaque, G. Rupak, H.W. Griesshammer, and H.W. Hammer, nucl-th/0207034.

[42] O. Zimmer (private communication).

[43] W.M. Snow (private communication).

[44] O. Yakubovsky, Sov. J. Nucl. Phys. 5, 937 (1967).

[45] A. Nogga, H. Kamada, and W. Glöckle, Phys. Rev. Lett. 85, 944 (2000).

[46] J.A. Tjon, Phys. Lett. B 56, 217 (1975).

[47] A. Kievsky (private communication).

[48] A. Kievsky, Phys. Rev. C 60, 034001 (1999).

[49] Y. Koike and J. Haidenbauer, Nucl. Phys. A463, 365c (1987).

[50] H. Witała, D. Hüber, and W. Glöckle, Phys. Rev. C 49, R14 (1994).

[51] H. Witała, W. Glöckle, J. Golak, A. Nogga, H. Kamada, R. Skibiński, and J. Kuroś-Żołnierczuk, Phys. Rev. C 63, 024007 (2001).

[52] E. Epelbaum, H. Kamada, A. Nogga, H. Witała, W. Glöckle, and Ulf-G. Meißner, Nucl. Phys. A689, 111 (2001).

[53] A. Kievsky, M. Viviani, S. Rosati, D. Hüber, W. Glöckle, H. Kamada, H. Witała, and J. Golak, Phys. Rev. C 58, 3085 (1998).

[54] W. Tornow, H. Witała, and A. Kievsky, Phys. Rev. C 57, 555 (1998).

[55] A. Kievsky, M. Viviani, and S. Rosati, Phys. Rev. C 64, 024002 (2001).

[56] J. Kuroś-Żołnierczuk, H. Witała, J. Golak, H. Kamada, A. Nogga, R. Skibiński, and W. Glöckle, Phys. Rev. C 66, 024003 (2002).

[57] J. Kuroś-Żołnierczuk, H. Witała, J. Golak, H. Kamada, A. 
Nogga, R. Skibiński, and W. Glöckle, Phys. Rev. C 66, 024003 (2002).

[58] Z. Zhou et al., Nucl. Phys. A684, 545c (2001).

[59] Y. Tachikawa, T. Yagita, S. Minami, T. Ishida, K. Tsuruta, and K. Sagara, Nucl. Phys. A684, 583c (2001).

[60] C. Howell et al., Nucl. Phys. A631, 692c (1998).

[61] P. Navrátil, J.P. Vary, W.E. Ormand, and B.R. Barrett, Phys. Rev. Lett. 87, 172502 (2001).

[62] P. Navrátil and W.E. Ormand, Phys. Rev. Lett. 88, 152502 (2002).

[63] S. Shimizu et al., Phys. Rev. C 52, 1193 (1995).

[64] K. Sagara et al., Phys. Rev. C 50, 576 (1994).

[65] J. McAninch, W. Haeberli, H. Witała, W. Glöckle, and J. Golak, Phys. Lett. B 307, 13 (1993).

[66] G. Rauprich et al., Few-Body Syst. 5, 67 (1988).

[67] F. Sperisen et al., Nucl. Phys. A422, 81 (1984).

[68] C.R. Howell et al., Few-Body Syst. 2, 19 (1987).

[69] H. Witała et al., Few-Body Syst. 15, 67 (1993).

[70] G. Rauprich et al., Nucl. Phys. A535, 313 (1991).

[71] H.R. Setze et al., Phys. Lett. B 388, 229 (1996).

[72] J. Strate et al., Nucl. Phys. A501, 51 (1989).

[73] J. Zejma et al., Phys. Rev. C 55, 42 (1997).

[74] M. Allet et al., Phys. Rev. C 50, 602 (1994).

[75] K. Bodek, J. Golak, L. Jarczyk, St. Kistryn, J. Kuroś, J. Lang, A. Micherdzińska, O. Naviliat-Cuncic, R. Skibiński, J. Smyrski, M. Sokołowski, J. Sromicki, A. Strzałkowski, H. Witała, J. Zejma, and W. Zipper, Few-Body Syst. 30, 65 (2001).

[76] From the point of view of an effective field theory it makes little sense to include only the $2 \mathrm{~N}$ force and to omit the $3 \mathrm{NF}$ contributing at the same order. In [10] we followed however the common trend in the field of few-nucleon physics and cal- culated various $3 N$ observables based on our NNLO $2 N$ potential for illustrative purposes. Such comparisons are helpful to identify the observables and kinematics most sensitive to the $3 \mathrm{NF}$ once a particular $2 \mathrm{NF}$ is specified.

[77] Similar observation for the purely short-range part of the $3 \mathrm{NF}$ has been made by Bedaque et al. [20], while Stewart pointed out that the two OPE terms in the expressions for the $3 \mathrm{NF}$ published in [12] are not independent from each other. Since these statements do not appear in the literature in a complete form we decided to demonstrate this explicitly here.

[78] Such factors can be calculated from expressions of the 3NF. For example, the antisymmetrized expression of the third term in Eq. (8) is 3 times smaller than the one of the fourth term, which has two additional insertions of the Pauli spin matrices.

[79] In fact, it would also be sufficient to make an estimation of the isospin breaking effects based upon purely $2 \mathrm{~N}$ forces at the level of precision of NNLO.

[80] One should be careful by looking at results which appear in the literature and sometimes indicate quite a strong deviation from the Phillips line. Especially earlier calculations have often been performed using not phase-equivalent potentials and with restricted accuracy.

[81] This value of $c_{D}$ is excluded by the observed value of the doublet scattering length.

[82] Hüber et al. pointed out in [17] that $A_{y}$ is sensitive to the choice of $c_{D}$. This is not in contradiction with the above discussion. We also observed a sensitivity to independent variations in $c_{D}$ and $c_{E}$, which is, however, strongly reduced as soon as one requires that the triton $\mathrm{BE}$ be reproduced.

[83] Significant deviation from the data in the minimum of $T_{21}$ can be observed at $3 \mathrm{MeV}$ as well, if $n p$-force corrections are taken into account. 